\title{
Six-loop beta functions in general scalar theory
}

\author{
A. Bednyakov ${ }^{a, b, 1}$ and A. Pikelner ${ }^{a}$ \\ ${ }^{a}$ Joint Institute for Nuclear Research, \\ Joliot-Curie, 6, Dubna 141980, Russia \\ ${ }^{b}$ P.N. Lebedev Physical Institute of the Russian Academy of Sciences, \\ Leninskii pr., 5, Moscow 119991, Russia \\ E-mail: bednya@theor.jinr.ru, pikelner@theor.jinr.ru
}

\begin{abstract}
We consider general renormalizable scalar field theory and derive six-loop beta functions for all parameters in $d=4$ dimensions within the $\overline{\mathrm{MS}}$-scheme. We do not explicitly compute relevant loop integrals but utilize $\mathrm{O}(n)$-symmetric model counter-terms available in the literature. We consider dimensionless couplings and parameters with a mass scale, ranging from the trilinear self-coupling to the vacuum energy. We use obtained results to extend renormalization-group equations for several vector, matrix, and tensor models to the six-loop order. Also, we apply our general expressions to derive new contributions to beta functions and anomalous dimensions in the scalar sector of the Two-Higgs-Doublet Model.
\end{abstract}

KEywords: Renormalization Group, Global Symmetries, Higgs Physics

ArXiv ePrint: 2102.12832

\footnotetext{
${ }^{1}$ Corresponding author.
} 


\section{Contents}

1 Introduction 1

2 Details of calculation 3

3 From general results to specific models 4

3.1 Warming up with $\mathrm{O}(n)$-symmetric model 4

3.2 Matrix models 5

3.2.1 Real anti-symmetric field 6

$\begin{array}{lll}3.2 .2 & \mathrm{O}(n) \times \mathrm{O}(m) \text { model } & 7\end{array}$

$\begin{array}{lll}3.2 .3 & \text { Complex anti-symmetric field } & 10\end{array}$

$\begin{array}{lll}3.2 .4 & \mathrm{U}(n) \times \mathrm{U}(m) \text { model } & 11\end{array}$

3.2.5 Field in the adjoint representation of $\mathrm{SU}(n) \quad 12$

3.3 Higher rank $\mathrm{O}(n) \times \mathrm{O}(n) \times \mathrm{O}(n)$ tensor model 14

3.4 Two-Higgs Doublet Model 15

$\begin{array}{lll}4 \text { Conclusion } & 15\end{array}$

A Deriving dimensionful couplings RG with dummy field method $\quad 17$

\section{Introduction}

The renormalization group ( $\mathrm{RG}$ ) plays an essential role in high-energy physics and the theory of critical phenomena. In particle physics, one can use RG to re-sum specific radiative corrections making theory predictions valid in a wide range of energy scales. In the study of critical phenomena, the RG approach allows one to study phase transitions and predict critical exponents of the second-order transitions with high accuracy.

A convenient tool to compute the RG functions that drive the dependence of model parameters on the scale is to use a perturbative expansion of dimensional regularized theory [1] together with modified minimal $\overline{\mathrm{MS}}$ subtraction of infinities. The latter appear in loop integrals and manifest themselves in $d$ dimensions as poles in $\varepsilon=(4-d) / 2$. One cancels the poles by a finite set of renormalization constants.

There is significant progress in the calculation of beta functions and anomalous dimensions in the $\overline{\mathrm{MS}}$ scheme. At the two-loop level, the RG functions are known in any general renormalizable quantum field theory (QFT) in $d=4$ dimensions [2-6]. Despite several calculations of three-loop (and even four-loop) RG functions in particular particle-physics models [7-17], general three-loop results are not yet available. Recently, an essential step has been made in this direction [18-21]. The main idea is to enumerate all possible "tensor" structures that can appear in the RG functions at a certain loop level and compute the 
corresponding unknown coefficients by matching them to specific models. Not long ago, this approach allowed authors of the paper [19] to derive general three-loop RG functions in a pure scalar model.

Our paper does not follow this strategy and extends the results for general scalar theories up to six loops by more conventional technique, i.e., by computing contributions from individual Feynman graphs. Such a leap in the loop level is due to the significant progress in calculating critical exponents in scalar theories. Thanks to the authors of ref. [22], the required renormalization constants can be found given the diagram-by-diagram results of the $\mathrm{KR}^{\prime}$ operation. Application of the latter to a Feynman graph produces the corresponding counter-term in the $\overline{\mathrm{MS}}$ scheme.

We consider the following general renormalizable Lagrangian

$$
\mathcal{L}=\frac{1}{2} \partial_{\mu} \phi_{a} \partial_{\mu} \phi_{a}-\frac{m_{a b}^{2}}{2} \phi_{a} \phi_{b}-\frac{h_{a b c}}{3 !} \phi_{a} \phi_{b} \phi_{c}-\frac{\lambda_{a b c d}}{4 !} \phi_{a} \phi_{b} \phi_{c} \phi_{d}-t_{a} \phi_{a}-\Lambda
$$

for real scalar fields $\phi_{a}$. The mass parameters $m_{a b}^{2}$, cubic $h_{a b c}$ and quartic couplings $\lambda_{a b c d}$ are symmetric in their indices. For completeness we also add the tadpole term proportional to $t_{a}$, and the vacuum energy term $\Lambda$.

Here we present the six-loop RG equations in the MS-scheme for the field $\phi_{a}$ and all parameters of eq. (1.1). The RG function for a parameter $A=\left\{\lambda_{a b c d}, h_{a b c}, m_{a b}^{2}, t_{a}, \Lambda\right\}$ is defined as

$$
\beta_{A} \equiv \mu \frac{\partial A}{\partial \mu}=\sum_{l} h^{l} \beta_{A}^{(l)}, \quad h=\frac{1}{16 \pi^{2}},
$$

where $\beta_{A}^{(l)}$ corresponds to the l-loop contribution. The field anomalous dimension is given by

$$
\phi_{a, 0}=Z_{a b} \phi_{b} \Rightarrow \gamma_{a b}^{\phi}=Z_{a c}^{-1} \cdot \mu \frac{\partial Z_{c b}}{\partial \mu}=-\mu \frac{\partial Z_{a c}^{-1}}{\partial \mu} \cdot Z_{c b}
$$

and is related to the field renormalization constant $Z_{a b}$. It is worth pointing that the latter can be multiplied by an arbitrary orthogonal matrix without spoiling divergence cancellation in two-point functions. Due to this, the antisymmetric part of $\gamma_{a b}^{\phi}$ is not fixed and leads to ambiguities ${ }^{1}$ in the RG functions. Nevertheless, the ambiguity is related to the freedom in the basis choice and does not affect physical observables (see discussions in refs. [12, 23]). In what follows we use symmetric $\gamma_{a b}^{\phi}$.

The paper is organized as follows. Section 2 contains details of our calculation. In section 3 we apply our general results to the cases known in the literature. In particular, we consider vector (section 3.1), matrix (section 3.2), and tensor (section 3.3) models possessing different kinds of symmetries. Also, we extend known three-loop results for the Two-Higgs-Doublet Model (2HDM) to six loops in section 3.4. Section 4 contains a discussion of the results and conclusions. In appendix A we provide a derivation of the RG functions for dimensionful couplings in a general form.

\footnotetext{
${ }^{1}$ We are grateful to F. Herren for bringing our attention to this fact.
} 


\section{Details of calculation}

As the calculation method, we decided to use an approach similar to the one in ref. [22], based on the direct computation of the necessary counter-terms from individual diagrams. However, in our work, we avoid the calculation of any loop integrals. The authors of ref. [22] considered all the required six-loop graphs in the context of the $\mathrm{O}(n)$-symmetric model $^{2}$ and made the corresponding counter terms available in a computer-readable form. One can adopt the latter for more complicated theories by changing model-dependent prefactors. In this way, six-loop renormalization-group functions for $\mathrm{O}(n)$ theory with cubic anisotropy [25] and $\mathrm{O}(n) \times \mathrm{O}(m)$ symmetric model [26] were derived.

To perform calculations with general Lagrangian (1.1), we prepare a DIANA [27] model file. We use special mapping rules between its internal topology format and diagram topologies, which are identified in ref. [22] and given in the Nickel index notation. After generating all needed two- and four-point functions with DIANA and performing all needed index contractions with FORM [28], we substitute actual values for momentum integrals by counter-terms from the available tables [22]. It is trivial to extract the RG functions $\gamma_{a b}^{\phi}$ and $\beta_{a b c d} \equiv \beta_{\lambda_{a b c d}}$ from the first $\varepsilon$ pole in the sum of counter-terms.

The obtained results involve a certain number of tensor structures, i.e., products of (up to 12) general couplings $\lambda_{a b c d}$ with all but four (two) indices contracted in $\beta_{a b c d}\left(\gamma_{a b}^{\phi}\right)$. We can simplify corresponding expressions by identifying tensor structures identical up to the renaming of contracted indices. Also, since the corresponding numeric coefficient depends only on the Feynman graph, we collect all the structures, which are different only by permutations of external indices $a b c d$. As a consequence, we can cast our main result for $\beta_{a b c d}$ into the form

$$
\beta_{a b c d}=\sum_{l=1}^{6} h^{l} \sum_{i}^{n_{l}} T_{i, a b c d}^{(l)} C_{i}^{(l)},
$$

where $n_{l}=\{1,2,7,23,110,571\}$ is the number of unique tensor structures $T_{i, a b c d}^{(l)}$ at $l$ loops. The coefficients $C_{i}^{(l)}$ are pure numbers. To deduce the expressions for $T_{i, a b c d}^{(l)}$, we made use of Nickel index notation [29] for graph representation of tensor contractions and utilized the GraphState package [30]. As an example, we give here one of the three-loop structures

$$
T_{4, a b c d}^{(3)} \equiv \frac{1}{4 !}\left[\lambda_{a i_{1} i_{2} i_{3}} \lambda_{b i_{3} i_{4} i_{6}} \lambda_{c i_{2} i_{4} i_{5}} \lambda_{d i_{1} i_{5} i_{6}}+\text { perm. }\right]=X, \quad \text { e123|e23|e3|e| }
$$

where we indicate the corresponding Nickel index and emphasize the normalization of $T_{i, a b c d}^{(l)}$ together with the fact that the latter are symmetric in $a b c d$.

We provide a table containing a minimal set of unique tensor structures formed by different contractions between $\lambda_{a b c d}$ indices and the corresponding coefficients. ${ }^{3}$ Given these tables, we derive the beta functions for dimensionful parameters entering (1.1) employing the so-called dummy field method [6,31,32]. The core of the technique is to introduce

\footnotetext{
${ }^{2}$ Seven-loop RG functions for the $\mathrm{O}(n)$ model are also known due to O. Schnetz [24].

${ }^{3}$ Examination of the structures contributing to $\gamma_{a b}^{\phi}$ leads us to the conclusion that in pure scalar theories the ambiguity in RG functions can manifests itself starting from five loops.
} 
"dummy" non-propagating field(s) $x_{a}$, e.g., by shifting all (or just one) components of the vector $\phi_{a} \rightarrow \phi_{a}+x_{a}$. Contracting $\beta_{a b c d}$ with one or more dummy fields $x_{a}$, we can readily obtain the expressions for $\beta_{\Lambda}, \beta_{a} \equiv \beta_{t_{a}}, \beta_{a b} \equiv \beta_{m_{a b}^{2}}$, and $\beta_{a b c} \equiv \beta_{h_{a b c}}$ (see appendix A). Indeed, we consider ${ }^{4} \beta_{x x x x}, \beta_{a x x x}, \beta_{a b x x}$, together with $\beta_{a b c x}$, and identify $\lambda_{a b c x} \equiv h_{a b c}$, $\lambda_{a b x x} \equiv 2 m_{a b}^{2}, \lambda_{a x x x} \equiv 3 ! t_{a}, \lambda_{x x x x} \equiv 4 ! \Lambda$. The only subtlety here is that we have to remove contributions from external leg renormalization, leading to tadpole diagrams in the final answer (see ref. [6] for details). We can immediately identify corresponding tensor structures in general expression for $\beta_{a b c d}$

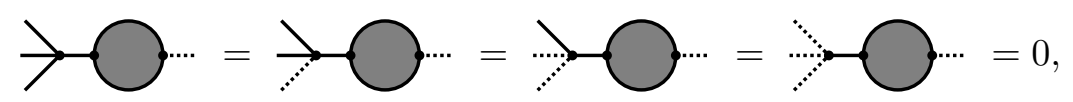

where dotted lines represent dummy field $x$. We use tilde to denote the quantities with tadpole contribution removed, and write

$$
\beta_{\Lambda}=\frac{1}{4 !} \cdot \tilde{\beta}_{x x x x}, \quad \beta_{a}=\frac{1}{3 !} \cdot \tilde{\beta}_{a x x x}, \quad \beta_{a b}=\frac{1}{2} \cdot \tilde{\beta}_{a b x x}, \quad \beta_{a b c}=\tilde{\beta}_{a b c x} .
$$

The tensor structures, including the corresponding graphs and coefficients for all the considered RG functions, can be found in the form of supplementary Mathematica files.

\section{From general results to specific models}

In this section we demonstrate the application of our general results to particular scalar models. It is worth mentioning that we heavily rely on FORM [28] to deal with index contractions and algebraic simplifications in the case of matrix fields.

\subsection{Warming up with $\mathrm{O}(n)$-symmetric model}

Our first example is the well-known $\mathrm{O}(n)$ symmetric model, which has a long history in the study of critical phenomena (see ref. [22] and reference therein). The following Euclidean Lagrangian describes the theory

$$
\mathcal{L}=\frac{1}{2} \vec{\phi}\left(-\partial^{2}+m^{2}\right) \vec{\phi}+\frac{\lambda}{4 !}(\vec{\phi} \cdot \vec{\phi})^{2}+\frac{1}{2} g_{\phi \phi} d_{a b} \phi_{a} \phi_{b},
$$

where $\vec{\phi}=\left\{\phi_{a}\right\}, a=1, \ldots, n$ is a $n$-component scalar field. We also add a quadratic operator involving traceless symmetric tensor $d_{a b}$ multiplied by a source $g_{\phi \phi}$. The anomalous dimension ${ }^{5} \gamma_{\phi \phi}$ of the corresponding operator is related to the so-called crossover exponent (see, e.g., refs. $[33,34]$ ) and can be found in our approach as

$$
\gamma_{\phi \phi}=-\beta_{g_{\phi \phi}}+2 \gamma_{\phi}
$$

with $\beta_{g_{\phi \phi}}$ being the beta function of $g_{\phi \phi}$ and $\gamma_{\phi}$ corresponding to the anomalous dimension of the field computed via eq. (1.3). This and other RG functions can be easily obtained

\footnotetext{
${ }^{4}$ We use compact notation $\beta_{x x x x} \equiv \beta_{a b c d} x_{a} x_{b} x_{c} x_{d}$, etc.

${ }^{5}$ In ref. [33] the notation $\gamma_{\tilde{\mathcal{E}}}=\gamma_{\phi \phi}$ is used.
} 
from our general result by means of substitutions

$$
\begin{aligned}
\lambda_{a b c d} & =\frac{\lambda}{3}\left(\delta_{a b} \delta_{c d}+\delta_{a c} \delta_{b d}+\delta_{a d} \delta_{b c}\right), \\
m_{a b}^{2} & =m^{2} \delta_{a b}+g_{\phi \phi} d_{a b} .
\end{aligned}
$$

In our calculation we find perfect agreement with previous computations. Our new result is related to the six-loop contribution to the beta function of the vacuum energy $\beta_{\Lambda}$ for $g_{\phi \phi}=0$ (see refs. [35, 36] for the five-loop expression). Using the notation $g \equiv h \lambda$ (cf. ref. [35]) we have ${ }^{6}$

$$
\begin{aligned}
\beta_{v} \equiv & \frac{1}{2} \cdot \frac{16 \pi^{2}}{m^{4}} \beta_{\Lambda} \\
= & \frac{n}{4}+\frac{n(n+2)}{96} g^{2}+\frac{n(n+2) g^{3}}{1296}(n+8)\left(12 \zeta_{3}-25\right) \\
& +\frac{n(n+2) g^{4}}{82944}\left[16 \zeta_{3}\left(3 n^{2}-382 n-1700\right)+96 \zeta_{4}\left(4 n^{2}+39 n+146\right)\right. \\
& \left.-1024 \zeta_{5}(5 n+22)-319 n^{2}+13968 n+64864\right] \\
& +\frac{n(n+2) g^{5}}{1866240}\left[384 \zeta_{3}^{2}\left(41 n^{2}-206 n-888\right)-960 \zeta_{6}\left(70 n^{2}+809 n+2118\right)\right. \\
& +96 \zeta_{5}\left(45 n^{3}+890 n^{2}+19348 n+67440\right)-288 \zeta_{4}\left(n^{3}+294 n^{2}+3088 n+9496\right) \\
& -48 \zeta_{3}\left(51 n^{3}+700 n^{2}-2964 n+2024\right)-1419 n^{3}-17124 n^{2}-2166136 n \\
& \left.-7308224+576 \zeta_{7}\left(67 n^{2}+1405 n+4306\right)+1152 \zeta_{3} \zeta_{4}\left(2 n^{2}+145 n+582\right)\right] .
\end{aligned}
$$

By simple rescaling $\lambda \rightarrow 3 ! \lambda$, one can easily get the six-loop contributions to the RG functions for the Standard Model Higgs potential parameters (including the vacuum energy) from the results of $\mathrm{O}(4)$ theory.

\subsection{Matrix models}

We consider matrix models with real and complex fields described by the following Lagrangians

$$
\mathcal{L}=\frac{1}{2} \operatorname{Tr}\left[\phi\left(-\partial^{2}+\tau\right) \phi^{T}\right]+\frac{\lambda_{1}}{4 !}\left(\operatorname{Tr}\left[\phi \phi^{T}\right]\right)^{2}+\frac{\lambda_{2}}{4 !} \operatorname{Tr}\left[\phi \phi^{T} \phi \phi^{T}\right]
$$

for real $\phi$ and

$$
\mathcal{L}=\operatorname{Tr}\left[\phi\left(-\partial^{2}+\tau\right) \phi^{\dagger}\right]+\frac{\lambda_{1}}{4 !}\left(\operatorname{Tr}\left[\phi \phi^{\dagger}\right]\right)^{2}+\frac{\lambda_{2}}{4 !} \operatorname{Tr}\left[\phi \phi^{\dagger} \phi \phi^{\dagger}\right]
$$

for complex $\phi$. To deal with matrix models we make use of the following decomposition (see also ref. [37])

$$
\phi=\sum_{a=1}^{N_{a}} \chi_{a} T_{a},
$$

\footnotetext{
${ }^{6}$ In ref. [35] RG functions are defined as derivatives w.r.t. $\ln \mu^{2}=2 \ln \mu$. The factor $\frac{1}{2}$ in (3.5) is introduced for convenience.
} 
where $\chi_{a}$ are real fields, and there are $N_{a}$ independent matrices $T_{a}$, which encode all the degrees of freedom present in $\phi$. Substituting (3.8) into either (3.6) or (3.7), we can rewrite the Lagrangians in the form (1.1). One can see that we completely get rid of the initial matrix indices of $\phi$ and replace them with a single one $a=1, \ldots, N_{a}$. Given eqs. (3.6) and (3.7), for the fields $\chi^{a}$ to be canonically normalized, we have to ensure that $\left[\left(T^{a}\right)^{\dagger} \equiv \bar{T}^{a}\right]$

$$
\begin{aligned}
\operatorname{Tr}\left(T_{a} T_{b}^{T}\right)+\operatorname{Tr}\left(T_{b} T_{a}^{T}\right) & =2 \delta_{a b}, & & \text { for real } \phi, \\
\operatorname{Tr}\left(T_{a} \bar{T}_{b}\right)+\operatorname{Tr}\left(T_{b} \bar{T}_{a}\right) & =\delta_{a b}, & & \text { for complex } \phi .
\end{aligned}
$$

As a consequence, one can identify

$$
\begin{aligned}
m_{a b}^{2} & =-\tau \delta_{a b}, \\
\lambda_{a b c d} & =\frac{\lambda_{1}}{4 !}\left[T^{a b} T^{c d}+\text { perm. }\right]+\frac{\lambda_{2}}{4 !}\left[T^{a b c d}+\text { perm. }\right],
\end{aligned}
$$

where

$$
\begin{array}{lll}
T^{a b}=\operatorname{Tr}\left(T_{a} T_{b}^{T}\right) \equiv T^{a b}, & T^{a b c d}=\operatorname{Tr}\left(T_{a} T_{b}^{T} T_{c} T_{d}^{T}\right) \equiv T^{a b c d}, & \text { for real } \phi, \\
T^{a b}=\operatorname{Tr}\left(T_{a} \bar{T}_{b}\right) \equiv T^{a b}, & T^{a b c d}=\operatorname{Tr}\left(T_{a} \bar{T}_{b} T_{c} \bar{T}_{d}\right) \equiv T^{a b c d}, & \text { for complex } \phi .
\end{array}
$$

In eq. (3.12) all 24 permutations of the indices $a b c d$ are taken into account. Obviously, the number of terms can be reduced in specific models. In the following subsections we provide some details of our calculations for the cases discussed in the literature.

\subsubsection{Real anti-symmetric field}

The Lagrangian of the model is given by eq. (3.6) with $\phi$ being an antisymmetric $n \times n$ matrix, $\phi^{T}=-\phi$. The model was considered in refs. [38, 39] and the four-loop results can be found in ref. [40].

To use our general formulae, we utilize the decomposition (3.8) with $N_{a}=\frac{n(n-1)}{2}$ and $T_{a}=t_{a}$ corresponding to antisymmetric generators of $\mathrm{SO}(n)$. The latter satisfy

$$
\operatorname{Tr}\left(t^{a} t^{b}\right)=T_{f} \delta_{a b}, \quad t_{i j}^{a} t_{k l}^{a}=\frac{T_{f}}{2}\left(\delta_{i l} \delta_{j k}-\delta_{i k} \delta_{j l}\right) .
$$

To keep the standard normalization for the fields $\chi_{a}$, we use $T_{f}=1$ (see eq. (3.9)). The number of terms in eq. (3.12) can be reduced ( $\left.\operatorname{Tr} t^{a} t^{b} \ldots \equiv T^{a b \ldots}\right)$

$$
\lambda_{a b c d}=\frac{\lambda_{1}}{3}\left[T^{a b} T^{c d}+T^{a c} T^{b d}+T^{a d} T^{b c}\right]+\frac{\lambda_{2}}{3}\left[T^{a b c d}+T^{a b d c}+T^{a c b d}\right],
$$

where we used the cyclic symmetry of the trace operation and the fact that $t_{a}^{T}=-t_{a}$.

By means of eq. (3.15) we write down the rules, which allow one to simplify the products of traces involving $t_{a}$ with some of the indices contracted. Substituting (3.16) into the general expression for $\beta_{a b c d}$, and performing the above-mentioned algebraic simplifications, we obtain $\beta_{a b c d}$ of the form

$$
4 ! \beta_{a b c d}=f_{1}\left(\lambda_{1}, \lambda_{2}, n\right)\left[T^{a b} T^{c d}+\text { perm. }\right]+f_{2}\left(\lambda_{1}, \lambda_{2}, n\right)\left[T^{a b c d}+\text { perm. }\right],
$$


where $f_{1,2}\left(\lambda_{1}, \lambda_{2}, n\right)$ are some polynomials of their arguments. It is possible to extract the beta functions for $\lambda_{1}$ and $\lambda_{2}$ from eq. (3.17) by applying suitable projectors. However, one can also use the fact that by construction $\beta_{a b c d}$ is symmetric in all the indices. Setting the latter equal to each other in the end of calculation, we have

$$
\beta_{\text {aaaa }}=f_{1}\left(\lambda_{1}, \lambda_{2}, n\right)\left[T^{a a}\right]^{2}+f_{2}\left(\lambda_{1}, \lambda_{2}, n\right)\left[T^{a a a a}\right], \quad \text { no sum over } a .
$$

Comparing eqs. (3.18) and (3.16) with $b=c=d=a$, one can easily deduce that

$$
\beta_{\lambda_{1}}=f_{1}\left(\lambda_{1}, \lambda_{2}, n\right), \quad \beta_{\lambda_{2}}=f_{2}\left(\lambda_{1}, \lambda_{2}, n\right) .
$$

We utilize this approach to obtain relevant RG functions up to the six-loop level. Our results agree with that given in refs. $[39,40] .{ }^{7}$ It is worth noting that for $n=2$ and $n=3$ the model is equivalent to one-component $\phi^{4}$ and the $\mathrm{O}(3)$-vector theory considered in section 3.1, respectively. Indeed, combining $\lambda=\lambda_{1}+\frac{1}{2} \lambda_{2}$ and computing $\beta_{\lambda}=\beta_{\lambda_{1}}+\frac{1}{2} \beta_{\lambda_{2}}$ for $n=2$ and $n=3$ we get the expected results.

Full six-loop beta functions and anomalous dimensions are available online as supplementary material. For convenience, we present here our expressions for the one-loop

$$
\begin{aligned}
& \beta_{\lambda_{1}}^{(1)}=\frac{\lambda_{1}^{2}}{6}\left(n^{2}-n+16\right)+\frac{\lambda_{1} \lambda_{2}}{3}(2 n-1)+\frac{\lambda_{2}^{2}}{2}, \\
& \beta_{\lambda_{2}}^{(1)}=4 \lambda_{1} \lambda_{2}+\frac{\lambda_{2}^{2}}{6}(2 n-1), \\
& \gamma_{\tau}^{(1)} \equiv-\beta_{\tau}^{(1)} / \tau=-\frac{\lambda_{1}}{6}\left(n^{2}-n+4\right)-\frac{\lambda_{2}}{6}(2 n-1),
\end{aligned}
$$

and two-loop RG functions

$$
\begin{aligned}
& \gamma_{\phi}^{(2)}=\frac{1}{288}\left[4 \lambda_{1}^{2}+\lambda_{2}^{2}\right]\left(n^{2}-n+4\right)+\frac{\lambda_{1} \lambda_{2}}{36}(2 n-1), \\
& \beta_{\lambda_{1}}^{(2)}=\frac{\lambda_{1}^{3}}{6}\left(3 n-3 n^{2}-28\right)-\left[\frac{11 \lambda_{1}^{2} \lambda_{2}}{9}+\frac{\lambda_{2}^{3}}{12}\right](2 n-1)-\frac{\lambda_{1} \lambda_{2}^{2}}{72}\left(5 n^{2}-5 n+164\right), \\
& \beta_{\lambda_{2}}^{(2)}=\frac{\lambda_{1}^{2} \lambda_{2}}{18}\left(5 n-5 n^{2}-164\right)-\frac{11 \lambda_{1} \lambda_{2}^{2}}{9}(2 n-1)+\frac{\lambda_{2}^{3}}{24}\left(n-n^{2}-20\right), \\
& \gamma_{\tau}^{(2)}=\frac{5}{144}\left[4 \lambda_{1}^{2}+\lambda_{2}^{2}\right]\left(n^{2}-n+4\right)+\frac{5 \lambda_{1} \lambda_{2}}{18}(2 n-1) .
\end{aligned}
$$

\subsection{2 $\mathrm{O}(n) \times \mathrm{O}(m)$ model}

Let us now consider a matrix model, which is invariant under $\mathrm{O}(n) \times \mathrm{O}(m)$ group. It describes the critical thermodynamics of frustrated spin systems with noncollinear and noncoplanar ordering (see, e.g., ref. [26] and references therein). In refs. [41] five-loop results are presented in terms of $u=\lambda_{1}+\lambda_{2}$, and $v=\lambda_{2}$. Six-loop RG functions are also known [26] in terms of $g_{i}=\lambda_{i}$.

The Landau-Wilson Lagrangian can be written in the form (3.6) with $\phi=\left\{\phi_{\alpha i}\right\}$ being $n \times m$ real matrix field, and $\alpha=1, \ldots, n, i=1, \ldots, m$.

\footnotetext{
${ }^{7}$ Note that in ref. [39] the notation $\lambda_{i}=g_{i}$ is used and the RG functions are expanded in $g_{i} /\left(8 \pi^{2}\right)$.
} 
To compute relevant RG functions from our general result we interpret $\chi_{a}$ in eq. (3.8) as $N_{a}=n \cdot m$ matrix elements of $\phi$, so that each of $n \times m$ real matrices $T^{a}$ has only one non-zero element

$$
\left(T_{a}\right)_{\alpha i}=\sqrt{T_{f}} \cdot \delta_{\alpha,((a-1) \operatorname{div} m)+1} \delta_{i,((a-1) \bmod m)+1},
$$

where we introduce $T_{f}=1$ for convenience. As a consequence, ${ }^{8}$ we have

$$
\operatorname{Tr}\left(T_{a} T_{b}^{T}\right)=\operatorname{Tr}\left(T_{b}^{T} T_{a}\right)=T_{f} \delta_{a b}, \quad T_{\alpha i}^{a} T_{\beta j}^{a}=T_{f} \delta_{\alpha \beta} \delta_{i j} .
$$

The quartic self-coupling is given by

$$
\begin{aligned}
\lambda_{a b c d}= & \frac{\lambda_{1}}{3}\left[T^{a b} T^{c d}+T^{a c} T^{b d}+T^{a d} T^{b c}\right] \\
& +\frac{\lambda_{2}}{6}\left[T^{a b c d}+T^{a b d c}+T^{a c b d}+T^{a c d b}+T^{a d b c}+T^{a d c b}\right],
\end{aligned}
$$

where to reduce the number of terms in l.h.s., we use the fact that

$$
\operatorname{Tr}(A)=\operatorname{Tr}\left(A^{T}\right), \quad\left(T_{a} T_{b}^{T} T_{c} T_{d}^{T}\right)^{T}=T_{d} T_{c}^{T} T_{b} T_{a}^{T}
$$

so

$$
T^{a b c d} \equiv \operatorname{Tr}\left(T_{a} T_{b}^{T} T_{c} T_{d}^{T}\right)=\operatorname{Tr}\left(T_{d} T_{c}^{T} T_{b} T_{a}^{T}\right) \equiv T^{d c b a} .
$$

To extract the RG functions, we substitute (3.29) together with (3.11) into $\beta_{a b c d}, \beta_{a b}$ and $\gamma_{a b}$ and use the rules (3.28) to simplify the products of traces involving $T_{a}$ and $T_{b}^{T}$.

We use known results $[26,41]$ to cross-check our expressions, which at the one-loop order are given by

$$
\begin{aligned}
& \beta_{\lambda_{1}}^{(1)}=\frac{\lambda_{1}^{2}}{3}(8+n m)+\frac{2 \lambda_{1} \lambda_{2}}{3}(1+n+m)+\lambda_{2}^{2}, \\
& \beta_{\lambda_{2}}^{(1)}=4 \lambda_{1} \lambda_{2}+\frac{\lambda_{2}^{2}}{3}(4+m+n), \\
& \gamma_{\tau}^{(1)} \equiv-\beta_{\tau}^{(1)} / \tau=-\frac{\lambda_{1}}{3}(2+n m)-\frac{\lambda_{2}}{3}(1+m+n),
\end{aligned}
$$

while at two loops we have

$$
\begin{aligned}
\gamma_{\phi}^{(2)}= & \frac{\lambda_{1}^{2}}{36}(2+m n)+\frac{\lambda_{1} \lambda_{2}}{18}(1+m+n)+\frac{\lambda_{2}^{2}}{72}(3+m n+m+n), \\
\beta_{\lambda_{1}}^{(2)}= & -\frac{\lambda_{1}^{3}}{3}(14+3 m n)-\frac{22 \lambda_{1}^{2} \lambda_{2}}{9}(1+m+n) \\
& -\frac{\lambda_{1} \lambda_{2}^{2}}{18}(87+5(m n+m+n))-\frac{\lambda_{3}^{2}}{3}(3+m+n), \\
\beta_{\lambda_{2}}^{(2)}= & -\frac{\lambda_{1}^{2} \lambda_{2}}{9}(82+5 m n)-\frac{2 \lambda_{1} \lambda_{2}^{2}}{9}(11(n+m)+29)-\frac{\lambda_{2}^{3}}{6}(17+m n+3(m+n)), \\
\gamma_{\tau}^{(2)}= & \frac{5 \lambda_{1}^{2}}{18}(2+m n)+\frac{5 \lambda_{1} \lambda_{2}}{9}(1+m+n)+\frac{5 \lambda_{2}^{2}}{36}(3+m n+m+n) .
\end{aligned}
$$

\footnotetext{
${ }^{8}$ Notice here that $T_{a} T_{b}^{T}$ are $n \times n$ matrices, while $T_{a}^{T} T_{b}$ have $m \times m$ dimension.
} 
In addition, we extend to the six-loop order the anomalous dimensions of quadratic operators considered in refs. [42, 43]:

$$
\begin{aligned}
Q_{\alpha i \beta j}^{(1)} & =\phi_{\alpha i} \phi_{\beta j}-\phi_{\alpha j} \phi_{\beta i}, \\
Q_{\alpha i \beta j}^{(2)} & =\frac{1}{2}\left(\phi_{\alpha i} \phi_{\beta j}+\phi_{\alpha j} \phi_{\beta i}\right)-\frac{1}{n} \delta_{\alpha \beta} \phi_{\delta i} \phi_{\delta j}-\frac{1}{m} \delta_{i j} \phi_{\alpha k} \phi_{\beta k}+\frac{1}{n m} \delta_{\alpha \beta} \delta_{i j} \phi_{\delta k} \phi_{\delta k}, \\
Q_{i j}^{(3)} & =\phi_{\delta i} \phi_{\delta j}-\frac{1}{m} \delta_{i j} \phi_{\delta k} \phi_{\delta k} \equiv \tilde{Q}_{\alpha \beta i j}^{(3)} \delta_{\alpha \beta}, \\
Q_{\alpha \beta}^{(4)} & =\phi_{\alpha k} \phi_{\beta k}-\frac{1}{n} \delta_{\alpha \beta} \phi_{\delta k} \phi_{\delta k} \equiv \tilde{Q}_{\alpha \beta i j}^{(4)} \delta_{i j}
\end{aligned}
$$

and belonging to different representations of $\mathrm{O}(n) \times \mathrm{O}(m)$. The operators can be treated in our approach in a similar fashion. We assume that the perturbations can be added to the Lagrangian with the corresponding sources ("masses") and rewritten in terms of $\chi$-fields as, e.g.,

$$
\begin{aligned}
{\left[\tilde{m}_{1, \alpha i \beta j}^{2}\right]\left[Q_{\alpha i \beta j}^{(1)}\right] } & =\left[\tilde{m}_{1, c d}^{2} T_{\alpha i}^{c} T_{\beta j}^{d}\right]\left[\chi^{a} \chi^{b}\left(T_{\alpha i}^{a} T_{\beta j}^{b}-T_{\alpha j}^{a} T_{\beta j}^{b}\right)\right] \equiv m_{1, a b}^{2} \chi^{a} \chi^{b} \\
m_{1, a b}^{2} & =\frac{1}{2} \tilde{m}_{1, c d}^{2}\left[T^{a c} T^{b d}-T^{a d b c}+(a \leftrightarrow b)\right]
\end{aligned}
$$

Since the operators (3.41) do not mix under renormalization, we use the following substitutions $^{9}$

$$
\begin{aligned}
Q_{\alpha i \beta j}^{(1)}: & m_{a b}^{2} \Rightarrow \frac{\tilde{m}_{1, c d}^{2}}{2}\left[T^{a c} T^{b d}-T^{a d b c}+(a \leftrightarrow b)\right], \\
Q_{\alpha i \beta j}^{(2)}: & m_{a b}^{2} \Rightarrow \frac{\tilde{m}_{2, c d}^{2}}{4}\left[T^{a c} T^{b d}+T^{a d b c}-\frac{2}{n} T^{a c d b}\right. \\
& \left.-\frac{2}{m} T^{a b c d}+(a \leftrightarrow b)\right]+\frac{\tilde{m}_{2, c d}^{2}}{n m} T^{a b} T^{c d}, \\
Q_{i j}^{(3)}: & m_{a b}^{2} \Rightarrow \tilde{m}_{3, c d}^{2}\left[\frac{1}{2}\left[T^{a c d b}+(a \leftrightarrow b)\right]-\frac{1}{m} T^{a b} T^{c d}\right], \\
Q_{\alpha \beta}^{(4)}: & m_{a b}^{2} \Rightarrow \tilde{m}_{4, c d}^{2}\left[\frac{1}{2}\left[T^{a b c d}+(a \leftrightarrow b)\right]-\frac{1}{n} T^{a b} T^{c d}\right]
\end{aligned}
$$

and extract the beta functions $\left(\beta_{\tilde{m}_{i}^{2}}\right)_{c d} \equiv-\tilde{\gamma}_{i} \cdot \tilde{m}_{i, c d}^{2}$ of $\tilde{m}_{i, c d}^{2}, i=1,4$ from the corresponding terms in $\beta_{a b}$. The RG functions for the operators $Q^{(i)}$ (3.41) are obtained by adding the contribution from the field anomalous dimension $\gamma_{\chi}=\gamma_{\phi}$ :

$$
\gamma_{Q_{i}}=\tilde{\gamma}_{i}+2 \gamma_{\phi}
$$

A welcome check of the result is the fact that for $v=0$ all $\gamma_{Q_{i}}$ coincide. We also compare our expressions with that given in ref. [43] and find perfect agreement up to five loops. ${ }^{10}$

\footnotetext{
${ }^{9}$ Given eq. (3.30), one can prove that tensors multiplying $\tilde{m}_{i, c d}^{2}$ are symmetric in $a \leftrightarrow b$ and $c \leftrightarrow d$.

${ }^{10}$ The results of ref. [43] are written in terms of $(u, v) /\left(8 \pi^{2}\right)$.
} 
We present here our one-loop,

$$
\begin{aligned}
\gamma_{Q_{1}}^{(1)} & =-\frac{2 \lambda_{1}-\lambda_{2}}{3}, \\
\gamma_{Q_{2}}^{(1)} & =-\frac{2 \lambda_{1}+\lambda_{2}}{3}, \\
\gamma_{Q_{3}}^{(1)} & =-\frac{2 \lambda_{1}+(1+n) \lambda_{2}}{3}, \\
\gamma_{Q_{4}}^{(1)} & =-\frac{2 \lambda_{1}+(1+m) \lambda_{2}}{3},
\end{aligned}
$$

and two-loop results

$$
\begin{aligned}
& \gamma_{Q_{1}}^{(2)}=\frac{\lambda_{1}^{2}}{9}(6+m n)+\frac{2 \lambda_{1} \lambda_{2}}{9}(1+m+n)+\frac{\lambda_{2}^{2}}{18}(1-m-n), \\
& \gamma_{Q_{2}}^{(2)}=\frac{\lambda_{1}^{2}}{9}(6+m n)+\frac{2 \lambda_{1} \lambda_{2}}{9}(3+m+n)+\frac{\lambda_{2}^{2}}{18}(9+m+n), \\
& \gamma_{Q_{3}}^{(2)}=\frac{\lambda_{1}^{2}}{9}(6+m n)+\frac{2 \lambda_{1} \lambda_{2}}{9}(3+m+3 n)+\frac{\lambda_{2}^{2}}{18}(9+m n+m+3 n), \\
& \gamma_{Q_{4}}^{(2)}=\frac{\lambda_{1}^{2}}{9}(6+m n)+\frac{2 \lambda_{1} \lambda_{2}}{9}(3+3 m+n)+\frac{\lambda_{2}^{2}}{18}(9+m n+3 m+n) .
\end{aligned}
$$

The six-loop expressions are available as supplementary material.

\subsubsection{Complex anti-symmetric field}

Let us now generalize the model discussed in section 3.2.1 and consider complex antisymmetric $n \times n$ matrices $\phi$. The corresponding Lagrangian (3.7) can be used to study phase transitions in quantum Fermi systems within the RG approach (see ref. [44]). We decompose the field via (3.8) with $N_{a}=n(n-1)$ and antisymmetric

$$
T^{a}= \begin{cases}t^{a} & a=1, \ldots, n(n-1) / 2, \\ i t^{a} & a=1+n(n-1) / 2, \ldots, n(n-1) .\end{cases}
$$

The latter are written in terms of generators $t^{a}$ of $\operatorname{SO}(n)$. Given $\operatorname{Tr}\left(t^{a} t^{b}\right)=T_{f} \delta_{a b}$, one can derive $\left[\left(T^{a}\right)^{\dagger} \equiv \bar{T}^{a}\right]$

$$
\begin{gathered}
\operatorname{Tr}\left(T^{a} \bar{T}^{b}\right)+\operatorname{Tr}\left(T^{b} \bar{T}^{a}\right)=-2 T_{f} \delta_{a b}, \quad T_{i j}^{a} T_{k l}^{a}=\sum_{b=1}^{N_{a} / 2}\left(t_{i j}^{b} t_{i j}^{b}+i^{2} t_{i j}^{b} t_{i j}^{b}\right)=0, \\
T_{i j}^{a} \bar{T}_{k l}^{a}=\sum_{b}^{N_{a} / 2}\left(t_{i j}^{b}\left(-t_{k l}^{b}\right)+i t_{i j}^{b}\left(+i t_{k l}^{b}\right)\right)=-T_{f}\left(\delta_{i l} \delta_{j k}-\delta_{i k} \delta_{j l}\right) .
\end{gathered}
$$

One can see from eq. (3.10) that for $T_{f}=-1 / 2$ the fields $\chi^{a}$ are canonically normalized. The self-coupling (3.12) is given by

$$
\begin{aligned}
\lambda_{a b c d}= & \frac{\lambda_{1}}{12}\left[T^{a b} T^{c d}+11 \text { permutations }\right] \\
& +\frac{\lambda_{2}}{6}\left[T^{a b c d}+T^{a b d c}+T^{a c b d} T^{c a d b}+T^{b a c d}+T^{b a d c}\right] .
\end{aligned}
$$


In writing the latter we take into account that

$$
\operatorname{Tr}(A)=\operatorname{Tr}\left(A^{T}\right), \quad\left(T^{a} \bar{T}^{b} T^{c} \bar{T}^{d}\right)^{T}=\left(T^{d}\right)^{*}\left(T^{c}\right)^{T}\left(T^{b}\right)^{*}\left(T^{a}\right)^{T}=\bar{T}^{d} T^{c} \bar{T}^{b} T^{a},
$$

so, e.g.,

$$
T^{a b c d} \equiv \operatorname{Tr}\left(T^{a} \bar{T}^{b} T^{c} \bar{T}^{d}\right)=\operatorname{Tr}\left(\bar{T}^{d} T^{c} \bar{T}^{b} T^{a}\right)=\operatorname{Tr}\left(T^{a} \bar{T}^{d} T^{c} \bar{T}^{b}\right) \equiv T^{a d c b}
$$

The expressions for the RG functions are available in literature up to the five-loop level $^{11}[45]$. We extend these results up to six loops. The one-loop contributions read

$$
\begin{aligned}
& \beta_{\lambda_{1}}^{(1)}=\frac{\lambda_{1}^{2}}{12}\left(n^{2}-n+8\right)+\frac{\lambda_{1} \lambda_{2}}{3}(n-1)+\frac{\lambda_{2}^{2}}{4}, \\
& \beta_{\lambda_{2}}^{(1)}=\lambda_{1} \lambda_{2}+\frac{\lambda_{2}^{2}}{12}(2 n-5), \\
& \gamma_{\tau}^{(1)} \equiv-\beta_{\tau}^{(1)} / \tau=-\frac{\lambda_{1}}{12}\left(n^{2}-n+2\right)-\frac{\lambda_{2}}{6}(n-1),
\end{aligned}
$$

while two-loop corrections are

$$
\begin{aligned}
& \gamma_{\phi}^{(2)}=\frac{\lambda_{1}^{2}}{576}\left(n^{2}-n-2\right)+\frac{\lambda_{1} \lambda_{2}}{144}(n-1)+\frac{\lambda_{2}^{2}}{1152}\left(n^{2}-3 n+4\right), \\
& \beta_{\lambda_{1}}^{(2)}=\frac{\lambda_{1}^{3}}{48}\left(3 n-3 n^{2}-14\right)-\frac{11 \lambda_{1}^{2} \lambda_{2}}{36}(n-1)+\frac{\lambda_{1} \lambda_{2}^{2}}{288}\left(15 n-5 n^{2}-92\right)-\frac{\lambda_{2}^{3}}{24}(n-2), \\
& \beta_{\lambda_{2}}^{(2)}=\frac{\lambda_{1}^{2} \lambda_{2}}{144}\left(5 n-5 n^{2}-82\right)+\frac{\lambda_{1} \lambda_{2}^{2}}{36}(20-11 n)+\frac{\lambda_{2}^{3}}{96}\left(7 n-n^{2}-20\right), \\
& \gamma_{\tau}^{(2)}=\frac{5 \lambda_{1}^{2}}{288}\left(n^{2}-n-2\right)+\frac{5 \lambda_{1} \lambda_{2}}{72}(n-1)+\frac{5 \lambda_{2}^{2}}{576}\left(n^{2}-3 n+4\right) .
\end{aligned}
$$

All results at six loops are available online as supplementary material.

\subsection{4 $\mathrm{U}(n) \times \mathrm{U}(m)$ model}

Consider now eq. (3.7) with general complex $n \times m$ matrix field $\phi=\left\{\phi_{\alpha i}\right\}$. The model can be used to study phase transitions in massless QCD and five-loop RG functions are available in literature [46]. We compute the six-loop contributions by means of decomposition (3.8) with $N_{a}=2 n m$ and $T^{a}$ being complex $n \times m$ matrices (cf. eq. (3.27))

$$
\left(T_{a}\right)_{\alpha i}=\sqrt{T_{f}} \begin{cases}\delta_{\alpha,((a-1) \operatorname{div} m)+1} \delta_{i,((a-1) \bmod m)+1}, & a=1, \ldots m n \\ i T_{\alpha i}^{a-m n}, & a=m n+1, \ldots 2 m n\end{cases}
$$

satisfying

$$
\operatorname{Tr}\left(T_{a} \bar{T}_{b}\right)+\operatorname{Tr}\left(T_{b} \bar{T}_{a}\right)=2 T_{f} \delta^{a b},
$$

and

$$
T_{\alpha j}^{a} T_{\beta k}^{a}=0, \quad \bar{T}_{j \alpha}^{a} \bar{T}_{k \beta}^{a}=0, \quad T_{\alpha j}^{a} \bar{T}_{k \beta}^{a}=2 T_{f} \delta_{\alpha \beta} \delta_{j k} .
$$

\footnotetext{
${ }^{11}$ In terms of $g_{i}=\lambda_{i} / 6$.
} 
The calculations are carried out with $T_{f}=1 / 2$ and the following representation of general self-coupling

$$
\lambda_{a b c d}=\frac{\lambda_{1}}{12}\left[T^{a b} T^{c d}+11 \text { perms }\right]+\frac{\lambda_{2}}{12}\left[T^{a b c d}+11 \text { perms }\right]
$$

where among all 24 permutations we exclude only those that correspond to the swapping between pairs of indices.

Our calculation employs eq. (3.67) and renders at one loop

$$
\begin{aligned}
& \beta_{\lambda_{1}}^{(1)}=\frac{\lambda_{1}^{2}}{6}(4+n m)+\frac{\lambda_{1} \lambda_{2}}{3}(n+m)+\frac{\lambda_{2}^{2}}{2}, \\
& \beta_{\lambda_{2}}^{(1)}=\lambda_{1} \lambda_{2}+\frac{\lambda_{2}^{2}}{6}(n+m), \\
& \gamma_{\tau}^{(1)} \equiv-\beta_{\tau}^{(1)} / \tau=-\frac{\lambda_{1}}{6}(1+n m)-\frac{\lambda_{2}}{6}(n+m),
\end{aligned}
$$

and at two loops

$$
\begin{aligned}
& \gamma_{\phi}^{(2)}=\frac{1}{288}\left(\lambda_{1}^{2}+\lambda_{2}^{2}\right)(1+m n)+\frac{\lambda_{1} \lambda_{2}}{144}(n+m), \\
& \beta_{\lambda_{1}}^{(2)}=-\frac{\lambda_{1}^{3}}{24}(7+3 m n)-\frac{11 \lambda_{1}^{2} \lambda_{2}}{36}(m+n)-\frac{\lambda_{1} \lambda_{2}^{2}}{72}(41+5 m n)-\frac{\lambda_{3}^{2}}{12}(m+n), \\
& \beta_{\lambda_{2}}^{(2)}=-\frac{\lambda_{1}^{2} \lambda_{2}}{72}(41+5 m n)-\frac{11 \lambda_{1} \lambda_{2}^{2}}{36}(m+n)-\frac{\lambda_{3}^{2}}{24}(5+m n), \\
& \gamma_{\tau}^{(2)}=\frac{5}{144}\left(\lambda_{1}^{2}+\lambda_{2}^{2}\right)(1+m n)+\frac{5 \lambda_{1} \lambda_{2}}{72}(n+m) .
\end{aligned}
$$

The full results are available as supplementary material. It is worth noting that for $m=n$ we get the four-loop results obtained in ref. [19] for the case of $\mathrm{U}(n) \times \mathrm{U}(n)$ model. ${ }^{12}$

\subsubsection{Field in the adjoint representation of $\mathrm{SU}(n)$}

In recent ref. [47] a model with $\phi$ being hermitian matrix field in the adjoint representation of $\mathrm{SU}(n)$ is analyzed both with perturbative and non-perturbative methods. In addition, the model was also considered as an example of application of the ARGES code [20]. We generalize the Lagrangian of ref. [47] and include also a cubic term ${ }^{13}$ (see also refs. [37, 48, 49]) together with the vacuum energy (we rescale $f$ and $\lambda_{2}$ for convenience)

$$
\mathcal{L}=\frac{1}{2} \operatorname{Tr}\left[\phi\left(-\partial^{2}+m^{2}\right) \phi\right]+\frac{\sqrt{n} f}{3 !} \operatorname{Tr} \phi^{3}+\frac{\lambda_{1}}{4}\left(\operatorname{Tr} \phi^{2}\right)^{2}+\frac{n \lambda_{2}}{4 !} \operatorname{Tr} \phi^{4}+\Lambda .
$$

Obviously, we can easily treat the model in our approach by means of the decomposition (3.8) with $T_{a}$ being SU( $\left.n\right)$ generators. The latter satisfy the well-known relations [50]

$$
\operatorname{Tr}\left(T_{a} T_{b}\right)=T_{f} \delta_{a b}, \quad T_{i j}^{a} T_{k l}^{a}=T_{f}\left(\delta_{i l} \delta_{k l}-\frac{1}{n} \delta_{i j} \delta_{k l}\right) .
$$

\footnotetext{
${ }^{12}$ In ref. [19] the RG functions are written in terms of $a_{u} \equiv h n \lambda_{2} / 24$ and $a_{v} \equiv h n^{2} \lambda_{1} / 24$.

${ }^{13}$ The term breaks $Z_{2}$ symmetry $\phi \rightarrow-\phi$ imposed in ref. [47].
} 
We utilize the normalization $T_{f}=1$ and substitute

$$
\begin{aligned}
\lambda_{a b c d}= & \frac{\lambda_{1}}{3}\left[T^{a b} T^{c d}+T^{a c} T^{b d}+T^{a d} T^{b c}\right] \\
& +\frac{n \lambda_{2}}{6}\left[T^{a b c d}+T^{a b d c}+T^{a c b d}+T^{a c d b}+T^{a d b c}+T^{a d c b}\right]
\end{aligned}
$$

together with

$$
h_{a b c}=\frac{\sqrt{n} f}{2}\left[T^{a b c}+T^{b a c}\right] .
$$

We obtain the RG functions up to the six-loop level, and at one loop we have

$$
\begin{aligned}
& \beta_{\lambda_{1}}^{(1)}=\frac{\lambda_{1}^{2}}{3}\left(7+n^{2}\right)+\frac{\lambda_{1} \lambda_{2}}{3}\left(4 n^{2}-6\right)+\lambda_{2}^{2}\left(3+n^{2}\right), \\
& \beta_{\lambda_{2}}^{(1)}=4 \lambda_{1} \lambda_{2}+\frac{\lambda_{2}^{2}}{3}\left(2 n^{2}-18\right), \\
& \beta_{f}^{(1)}=f\left[2 \lambda_{1}+\lambda_{2}\left(n^{2}-6\right)\right], \\
& \beta_{m^{2}}^{(1)}=\frac{m^{2}}{3}\left[\lambda_{1}\left(n^{2}+1\right)+\lambda_{2}\left(2 n^{2}-3\right)\right]+\frac{f^{2}}{2}\left(n^{2}-4\right), \\
& \beta_{\Lambda}^{(1)}=\frac{m^{4}}{2}\left(n^{2}-1\right) .
\end{aligned}
$$

The two-loop expressions are given by

$$
\begin{aligned}
\gamma_{\phi}^{(2)}= & \frac{\lambda_{1}^{2}}{36}\left(1+n^{2}\right)-\frac{\lambda_{1} \lambda_{2}}{18}\left(3-2 n^{2}\right)+\frac{\lambda_{2}^{2}}{72}\left(18-6 n^{2}+n^{4}\right), \\
\beta_{\lambda_{1}}^{(2)}= & -\frac{\lambda_{1}^{3}}{3}\left(11+3 n^{2}\right)+\frac{22 \lambda_{1}^{2} \lambda_{2}}{9}\left(3-2 n^{2}\right) \\
& -\frac{\lambda_{1} \lambda_{2}^{2}}{18}\left(306+42 n^{2}+5 n^{4}\right)+\frac{\lambda_{2}^{3}}{3}\left(36+9 n^{2}-2 n^{4}\right), \\
\beta_{\lambda_{2}}^{(2)}= & \frac{\lambda_{1}^{2} \lambda_{2}}{9}\left(77+5 n^{2}\right)+\frac{2 \lambda_{1} \lambda_{2}^{2}}{9}\left(123-22 n^{2}\right)-\frac{\lambda_{2}^{3}}{6}\left(174-16 n^{2}+n^{4}\right), \\
\beta_{f}^{(2)}= & \frac{f}{24}\left[4 \lambda_{1} \lambda_{2}\left(105-22 n^{2}\right)-2 \lambda_{1}^{2}\left(35+3 n^{2}\right)-\lambda_{2}^{2}\left(630-138 n^{2}+11 n^{4}\right)\right] \\
\beta_{m^{2}}^{(2)}= & \frac{5 m^{2}}{36}\left[4 \lambda_{1} \lambda_{2}\left(3-2 n^{2}\right)-2 \lambda_{1}^{2}\left(1+n^{2}\right)-\lambda_{2}^{2}\left(18-6 n^{2}+n^{4}\right)\right] \\
& +\frac{f^{2}}{12}\left[\lambda_{1}\left(36-5 n^{2}-n^{4}\right)-3 \lambda_{2}\left(36-17 n^{2}+2 n^{4}\right)\right], \\
\beta_{\Lambda}^{(2)}= & -\frac{m^{2} f^{2}}{4}\left(n^{2}-1\right)\left(n^{2}-4\right) .
\end{aligned}
$$

To compare our results with that of ref. [47], one has to take into account that the latter correspond to $f=0$ and are written in terms of $g_{i} /\left(8 \pi^{2}\right)$ with $g_{1}=\left(n \lambda_{2}\right) / 6$ and $g_{2}=\lambda_{1} / 6$. We also use the expressions obtained by means of ARGES [20] to cross-check $\gamma_{\phi}$ and the beta functions for $\lambda_{1}, \lambda_{2}$, and $m^{2}$ up to 4 loops. 


\subsection{Higher rank $\mathrm{O}(n) \times \mathrm{O}(n) \times \mathrm{O}(n)$ tensor model}

To give an example how to apply our general result to models with more complicated index structure, we consider evaluation of the beta functions in the model with $\mathrm{O}(n) \times \mathrm{O}(n) \times \mathrm{O}(n)$ symmetry [51]. The model Lagrangian is

$$
\mathcal{L}=\frac{1}{2} \partial_{\mu} \phi_{a b c} \partial_{\mu} \phi_{a b c}+\frac{\lambda_{1}}{4 !} T_{\phi_{1} \phi_{2} \phi_{3} \phi_{4}}^{\mathrm{t}}+\frac{\lambda_{2}}{4 !} T_{\phi_{1} \phi_{2} \phi_{3} \phi_{4}}^{\mathrm{p}}+\frac{\lambda_{3}}{4 !} T_{\phi_{1} \phi_{2} \phi_{3} \phi_{4}}^{\mathrm{ds}},
$$

where we follow the naming scheme from ref. [51] for the interaction terms as "tetrahedral", "pillow" and "double-sum". Again, we use fields as tensor indices of the structures $T^{i}$ to indicate contractions of triplets of indexes with $\phi_{a b c}$. For convenience, we present the tensor structures in the following pictorial form:

$$
T^{\mathrm{t}}=\frac{1}{3}\left(\begin{array}{c}
a_{1} \\
b_{1} \\
c_{1}
\end{array}\right.
$$

To map the model onto our general result, we associate open indices in general model (1.1) with multi index $i_{k}=\left\{a_{k}, b_{k}, c_{k}\right\}$, and rewrite the self-coupling (3.91) in the form:

$$
\lambda_{i_{1} i_{2} i_{3} i_{4}}=\lambda_{1} T_{\left(i_{1} i_{2} i_{3} i_{4}\right)}^{\mathrm{t}}+\lambda_{2} T_{\left(i_{1} i_{2} i_{3} i_{4}\right)}^{\mathrm{p}}+\lambda_{3} T_{\left(i_{1} i_{2} i_{3} i_{4}\right)}^{\mathrm{ds}}
$$

where $\left(i_{1} i_{2} i_{3} i_{4}\right)$ denotes symmetrization. At one loop we get

$$
\begin{aligned}
& \beta_{1}^{(1)}=\frac{2 \lambda_{1} \lambda_{2}}{3}(1+n)+4 \lambda_{1} \lambda_{3}+\frac{4 \lambda_{2}^{2}}{9} \\
& \beta_{2}^{(1)}=\lambda_{1}^{2}(2+n)+\frac{4 \lambda_{1} \lambda_{2}}{3}(2+n)+4 \lambda_{2} \lambda_{3}+\frac{\lambda_{2}^{2}}{9}\left(12+5 n+n^{2}\right), \\
& \beta_{3}^{(1)}=\frac{2 \lambda_{1} \lambda_{2}}{3}+2 \lambda_{1} \lambda_{3} n+\frac{2 \lambda_{2} \lambda_{3}}{3}\left(1+n+n^{2}\right)+\frac{\lambda_{2}^{2}}{9}(3+2 n)+\frac{\lambda_{3}^{2}}{3}\left(8+n^{3}\right) .
\end{aligned}
$$

while two-loop contribution renders

$$
\begin{aligned}
\beta_{1}^{(2)}= & \frac{\lambda_{1}^{3}}{18}\left(n^{3}-15 n-10\right)-\frac{2 \lambda_{1}^{2} \lambda_{2}}{9}\left(n^{2}+4 n+13\right)-\frac{10 \lambda_{1}^{2} \lambda_{3}}{3} n \\
& -\frac{\lambda_{1} \lambda_{2}^{2}}{54}\left(n^{3}+15 n^{2}+93 n+101\right)-\frac{2 \lambda_{1} \lambda_{2} \lambda_{3}}{9}\left(5 n^{2}+17 n+17\right) \\
& -\frac{\lambda_{1} \lambda_{3}^{2}}{9}\left(5 n^{3}+82\right)-\frac{2 \lambda_{2}^{3}}{81}\left(2 n^{2}+13 n+24\right)-\frac{16 \lambda_{2}^{2} \lambda_{3}}{9},
\end{aligned}
$$




$$
\begin{aligned}
\beta_{2}^{(2)}= & -\frac{2 \lambda_{1}^{3}}{3}\left(n^{2}+n+4\right)-\frac{\lambda_{1}^{2} \lambda_{2}}{18}\left(n^{3}+12 n^{2}+99 n+98\right)-4 \lambda_{1}^{2} \lambda_{3}(n+2) \\
& -\frac{2 \lambda_{1} \lambda_{2}^{2}}{9}\left(4 n^{2}+18 n+29\right)-\frac{2 \lambda_{1} \lambda_{2} \lambda_{3}}{3}(13 n+16)-\frac{\lambda_{2}^{3}}{162}\left(5 n^{3}+45 n^{2}+243 n+343\right) \\
& -\frac{2 \lambda_{2}^{2} \lambda_{3}}{9}\left(7 n^{2}+15 n+29\right)-\frac{\lambda_{2} \lambda_{3}^{2}}{9}\left(5 n^{3}+82\right), \\
\beta_{3}^{(2)}= & -\frac{\lambda_{1}^{3} n}{3}-\frac{2 \lambda_{1}^{2} \lambda_{2}}{9}\left(n^{2}+n+4\right)-\frac{5 \lambda_{1}^{2} \lambda_{3}}{18}\left(n^{3}+3 n+2\right)-\frac{8 \lambda_{1} \lambda_{2}^{2}}{9}(n+1) \\
& -\frac{2 \lambda_{1} \lambda_{2} \lambda_{3}}{9}\left(5 n^{2}+5 n+17\right)-\frac{22 \lambda_{1} \lambda_{3}^{2}}{3} n-\frac{7 \lambda_{2}^{3}}{81}\left(n^{2}+3 n+5\right) \\
& -\frac{\lambda_{2}^{2} \lambda_{3}}{54}\left(5 n^{3}+15 n^{2}+93 n+97\right)-\frac{22 \lambda_{2} \lambda_{3}^{2}}{9}\left(n^{2}+n+1\right)-\frac{\lambda_{3}^{3}}{3}\left(3 n^{3}+14\right) .
\end{aligned}
$$

Modulo rescaling $\lambda_{i}=6 g_{i}$, the obtained expressions coincide with those given in ref. [51]. Six-loop ${ }^{14}$ results can be found in the form of supplementary material.

\subsection{Two-Higgs Doublet Model}

Motivated by three-loop calculation [13] in 2HDM model (see, e.g., refs. [52, 53] for review), we consider the following general renormalizable Higgs potential

$$
\begin{aligned}
V_{2 \mathrm{HDM}}= & m_{11}^{2} \Phi_{1}^{\dagger} \Phi_{1}+m_{22}^{2} \Phi_{2}^{\dagger} \Phi_{2}-\left(m_{12}^{2} \Phi_{1}^{\dagger} \Phi_{2}+\text { h.c. }\right) \\
& +\frac{1}{2} \lambda_{1}\left(\Phi_{1}^{\dagger} \Phi_{1}\right)^{2}+\frac{1}{2} \lambda_{2}\left(\Phi_{2}^{\dagger} \Phi_{2}\right)^{2}+\lambda_{3}\left(\Phi_{1}^{\dagger} \Phi_{1}\right)\left(\Phi_{2}^{\dagger} \Phi_{2}\right)+\lambda_{4}\left(\Phi_{1}^{\dagger} \Phi_{2}\right)\left(\Phi_{2}^{\dagger} \Phi_{1}\right) \\
& +\left[\frac{1}{2} \lambda_{5}\left(\Phi_{1}^{\dagger} \Phi_{2}\right)^{2}+\lambda_{6}\left(\Phi_{1}^{\dagger} \Phi_{1}\right)\left(\Phi_{1}^{\dagger} \Phi_{2}\right)+\lambda_{7}\left(\Phi_{2}^{\dagger} \Phi_{2}\right)\left(\Phi_{1}^{\dagger} \Phi_{2}\right)+\text { h.c. }\right], \quad(3.101)
\end{aligned}
$$

where $\Phi_{1,2}$ are $\mathrm{SU}(2)$ doublets. The self-couplings $\lambda_{1-4}$ and the mass parameters $m_{11}^{2}$, $m_{22}^{2}$ are real, while $\lambda_{5-7}$, and $m_{12}^{2}$ can be complex. Due to the freedom in redefinition of Higgs-field basis, only 11 of 14 real parameters in eq. (3.101) are independent. In ref. [13] convenient variables $[52,53]$ and the so-called reparametrization invariants (see, e.g., ref. [54] for a comprehensive study) were used to compute the RG functions.

In this work, we use another strategy and directly calculate the beta function of $\lambda_{1-7}$ together with the anomalous dimensions of $m_{11}^{2}, m_{22}^{2}$, and $m_{12}^{2}$ from our general expressions. We enumerate all real components of two doublets $\Phi_{1,2}$ and rewrite eq. (3.101) in the general form (1.1) with indices $a, b$, etc. running from one to eight. We find full agreement with previous results and extend the latter up to six loops. We have checked that our expressions for $\beta_{\lambda_{2}}\left(\beta_{\lambda_{7}}\right)$ can be obtained from $\beta_{\lambda_{1}}\left(\beta_{\lambda_{6}}\right)$ via the replacement $\lambda_{1} \leftrightarrow \lambda_{2}$ and $\lambda_{6} \leftrightarrow \lambda_{7}$. One can use the same substitutions together with $m_{11}^{2} \leftrightarrow m_{22}^{2}$ to get $\beta_{m_{22}^{2}}$ from $\beta_{m_{11}^{2}}$. We make the six-loop results available as supplementary material.

\section{Conclusion}

We considered the general renormalizable scalar QFT model and directly computed the RG functions for the quartic and cubic self-couplings, mass parameter, tadpole term, and

\footnotetext{
${ }^{14}$ Due to the large number of indices in $\lambda_{i_{1} i_{2} i_{3} i_{4}}$ (3.94), we use the JINR supercomputer "Govorun" to compute the required 571 six-loop tensors in parallel.
} 
vacuum energy. In deriving our results for dimensionless quantities, we used the expressions for the $\mathrm{KR}^{\prime}$ operation applied to individual Feynman integrals. The latter are publicly available thanks to lengthy and non-trivial calculations of ref. [22]. To compute the RG functions of dimensionful parameters, we utilize the powerful dummy field technique.

To validate our general results, we considered several scalar models discussed in the theory of critical phenomena. We found perfect agreement with known results and extend them by computing several missing six-loop contributions. Among the latter are the vacuum energy beta function in the $\mathrm{O}(n)$ model, the anomalous dimensions of quadratic perturbations in the $\mathrm{O}(n) \times \mathrm{O}(m)$ model, and the self-coupling beta functions for $\mathrm{U}(n) \times \mathrm{U}(m)$, and $\mathrm{O}(n) \times \mathrm{O}(n) \times \mathrm{O}(n)$ models and the model with the Higgs field in the adjoint representation of the $\mathrm{SU}(n)$ group. Additionally, we extend the three-loop results for the general Two-Higgs-Doublet Model scalar sector to six loops.

We believe that the obtained state-of-the-art RG functions are of immediate interest to the condensed-matter community. On the contrary, present six-loop results can hardly find their applications in phenomenological analyses of the Standard Model extensions in the near future. However, it is convenient to estimate the influence of the high-order terms on extended Higgs sector studies, which currently rely on the two- or three-loop RG. Public codes for RG analyses [21, 37, 55-57] can be equipped with our results to carry out this kind of computations.

We also note that the expression for vacuum energy beta function is relevant for effective potential $V_{\text {eff }}(\phi)$ RG improvement (see, e.g., ref. [58]). Moreover, in recent ref. [59], the vacuum energy function's role is emphasized in the effective field theory approach to $V_{\text {eff }}(\phi)$ computation in models with many different scales.

Let us also mention here that seven-loop results [24] can not be directly used in our approach. We expect that in the future when the corresponding diagram-by-diagram counterterms will be available, one can almost immediately extend our general expressions to one more loop. However, our experience tells us that the calculation of tensor structures in specific models can be very time-consuming.

\section{Acknowledgments}

We thank G.Kalagov, M.Kompaniets, N.Lebedev, and F. Herren for fruitful discussions. We also thank T. Steudtner for the correspondence regarding refs. [19, 20] and sharing his four-loop results. We are grateful to the Joint Institute for Nuclear Research for letting us use their supercomputer "Govorun". The work of A.B. is supported by the Grant of the Russian Federation Government, Agreement No. 14.W03.31.0026 from 15.02.2018. The work of A.P. is supported by the Foundation for the Advancement of Theoretical Physics and Mathematics "BASIS." 

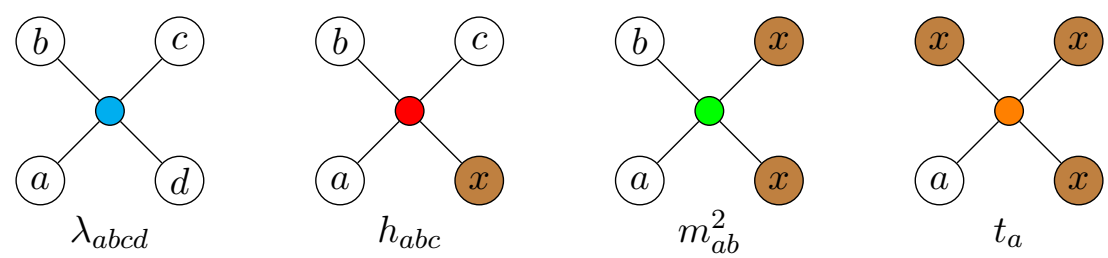

Figure 1. Representation of vertices corresponding to the parameters of the Lagrangian (1.1). The index " $x$ " denotes the contraction with a dummy field.

\section{A Deriving dimensionful couplings RG with dummy field method}

The author of ref. [19] introduced a convenient representation for four-loop quartic-coupling beta function with all the self-couplings involving external indices explicitly factorized. We adopt this ansatz to all loops

$$
\begin{aligned}
\beta_{a b c d}= & {\left[\lambda_{a b c f} \gamma_{f d}^{\phi}+\lambda_{a b d f} \gamma_{f c}^{\phi}+\lambda_{a c d f} \gamma_{f b}^{\phi}+\lambda_{b c d f} \gamma_{f a}^{\phi}\right]+\left[\lambda_{a b e f} \lambda_{c d g h} \bigcirc_{e f \mid g h}+5 \text { perm. }\right] } \\
& +\left[\lambda_{a b e f} \lambda_{c g h i} \lambda_{d j k l} \triangle_{e f|g h i| j k l}+11 \text { perm. }\right] \\
& +\left[\lambda_{a e f g} \lambda_{b h i j} \lambda_{c k l m} \lambda_{d n o p} \square_{e f g|h i j| k l m \mid n o p}+23 \text { perm. }\right]
\end{aligned}
$$

and re-derive the RG functions for the dimensionful parameters entering the Lagrangian (1.1). In eq. (A.1) "perm." denotes the terms, which can be obtained from the respective expressions via non-equivalent permutations of external indices. It is convenient to represent eq. (A.1) in the following pictorial form:

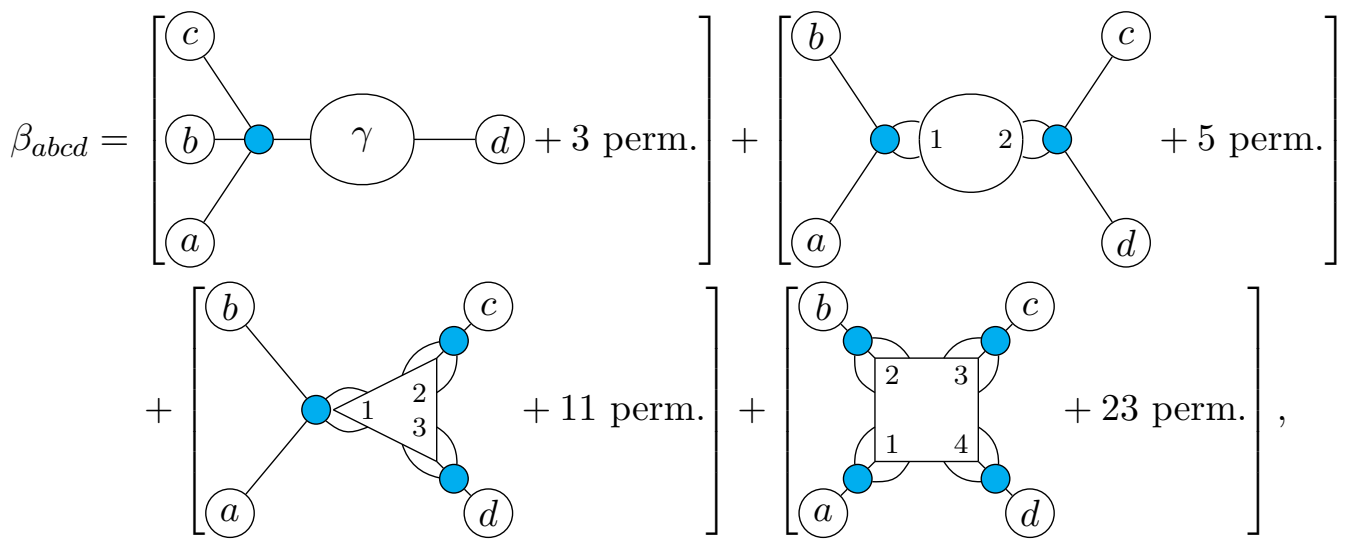

where external self-couplings are denoted by blue vertices (see figure 1) and we use the notation given in figure 2 for the non-external parts of four-point functions. It is worth noting that $\bigcirc_{a b \mid c d}, \triangle_{a b|c d e| f g h}$, and $\square_{a b c|d e f| g h i \mid j k l}$ do not need to be symmetric w.r.t. permutations of (group of) indices. Each group of indices is contracted with symmetric couplings, and, thus, does not need to be explicitly symmetrized. However, we explicitly take into account that an external index $a, b, c$, or $d$ can be attached to any group via a quartic vertex (this corresponds the permutations indicated, e.g., in eq. (A.2)). Due to this, we distinguish index groups and mark them by numbers (cf., figure 2). 


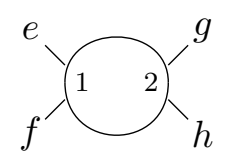

Oefigh

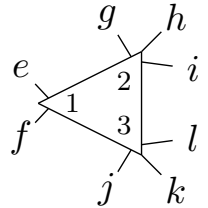

$\triangle_{e f|g h i| j k l}$

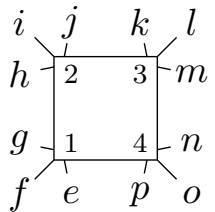

$\square_{e f g|h i j| k l m \mid n o p}$

Figure 2. Graphical representation of the structures entering $\beta_{a b c d}$ (A.1). The numbers encode the positions of index groups (separated by vertical lines) in the corresponding expressions.

Let us now contract the expression (A.2) with external dummy field $x_{d}$ and exclude the tadpole graphs discussed in section 2 :

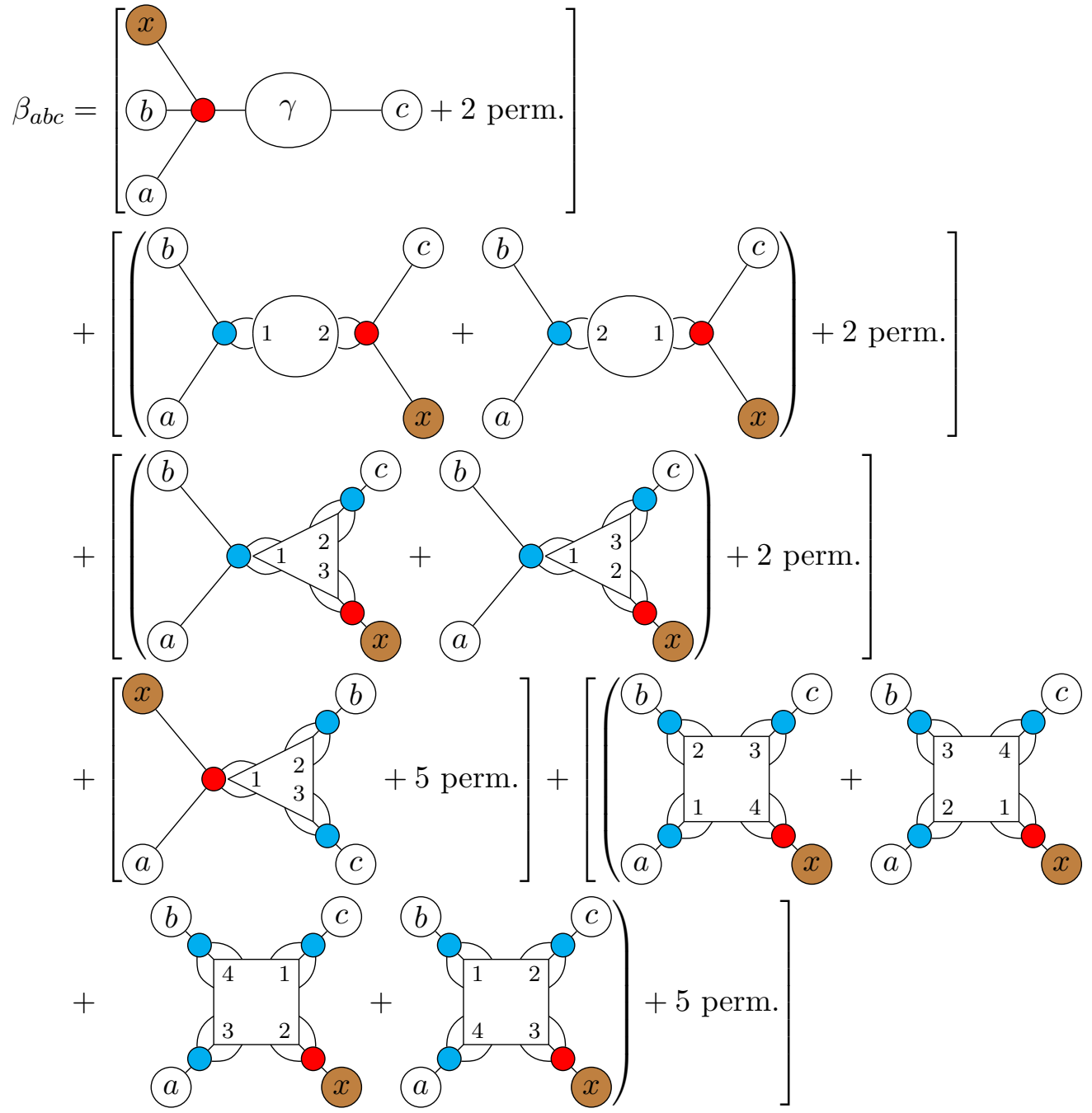

Here the trilinear couplings correspond to red vertices (see figure 1) and again we have to explicitly take into account permutations of external indices. The analytic expression is given by

$$
\begin{aligned}
\beta_{a b c}= & {\left[h_{a b f} \gamma_{f c}^{\phi}+h_{a c f} \gamma_{f b}^{\phi}+h_{b c f} \gamma_{f a}^{\phi}\right]+\left[\lambda_{a b e f} h_{c g h}\left(\bigcirc_{e f \mid g h}+\bigcirc_{g h \mid e f}\right)+2 \text { perm. }\right] } \\
& +\left[\lambda_{a b e f} \lambda_{c g h i} h_{j k l}\left(\triangle_{e f|g h i| j k l}+\triangle_{e f|j k l| g h i}\right)+2 \text { perm. }\right]
\end{aligned}
$$




$$
\begin{aligned}
& +\left[h_{a e f} \lambda_{b g h i} \lambda_{c j k l} \triangle_{e f|g h i| j k l}+5 \text { perm. }\right]+\left[\lambda _ { a e f g } \lambda _ { b h i j } \lambda _ { c k l m } h _ { n o p } \left(\square_{e f g|h i j| k l m \mid \text { nop }}\right.\right. \\
& \left.\left.+\square_{\text {nop } \mid \text { efg|hij|klm }}+\square_{k l m \mid \text { nop } \mid \text { efg } \mid \text { hij }}+\square_{\text {hij|klm|nop } \mid \text { efg }}\right)+5 \text { perm. }\right]
\end{aligned}
$$

To obtain the beta function for mass parameter we contract eq. (A.3) with one more dummy field $x_{c}$. Dividing the result by the factor of two, we get
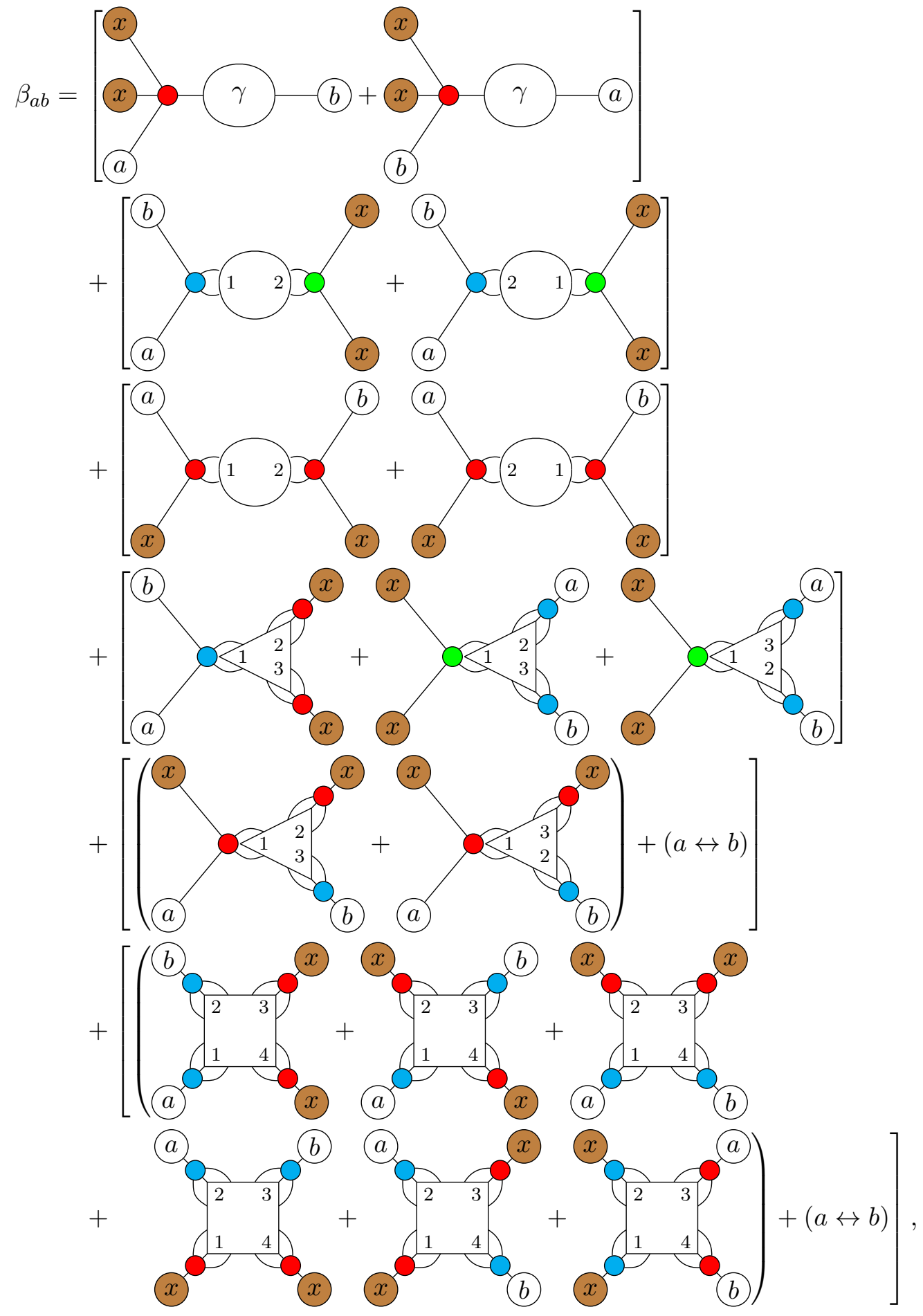
where red dots denote mass parameter $m_{a b}^{2}$ insertions (cf. figure 1). The corresponding analytic expression is given by ${ }^{15}$

$$
\begin{aligned}
\beta_{a b}= & {\left[m_{a f}^{2} \gamma_{f b}^{\phi}+m_{b f}^{2} \gamma_{f a}^{\phi}\right]+\left[\left(\lambda_{a b e f} m_{g h}^{2}+h_{a e f} h_{b g h}\right)\left(\bigcirc_{e f \mid g h}+\bigcirc_{g h \mid e f}\right)\right] } \\
& +\left[\lambda_{a b e f} h_{g h i} h_{j k l} \triangle_{e f|g h i| j k l}+m_{e f}^{2} \lambda_{a g h i} \lambda_{b j k l}\left(\triangle_{e f|g h i| j k l}+\triangle_{e f|j k l| g h i}\right)\right] \\
& {\left[h_{a e f} h_{g h i} \lambda_{b j k l}\left(\triangle_{e f|g h i| j k l}+\triangle_{e f|j k l| g h i}\right)+(a \leftrightarrow b)\right] } \\
& +\left[\lambda _ { a e f g } \lambda _ { b h i j } h _ { k l m } h _ { n o p } \left(\square_{e f g|h i j| k l m \mid n o p}+\square_{e f g|k l m| h i j \mid n o p}+\square_{e f g|k l m| n o p \mid h i j}\right.\right. \\
& \left.\left.+\square_{k l m|e f g| h i j \mid n o p}+\square_{k l m|e f g| n o p \mid h i j}+\square_{k l m|n o p| e f g \mid h i j}\right)+(a \leftrightarrow b)\right] .
\end{aligned}
$$

We proceed further and obtain the RG function for the tadpole term. Contracting eq. (A.5) with $x_{b}$ and dividing by the factor of 3 , we get

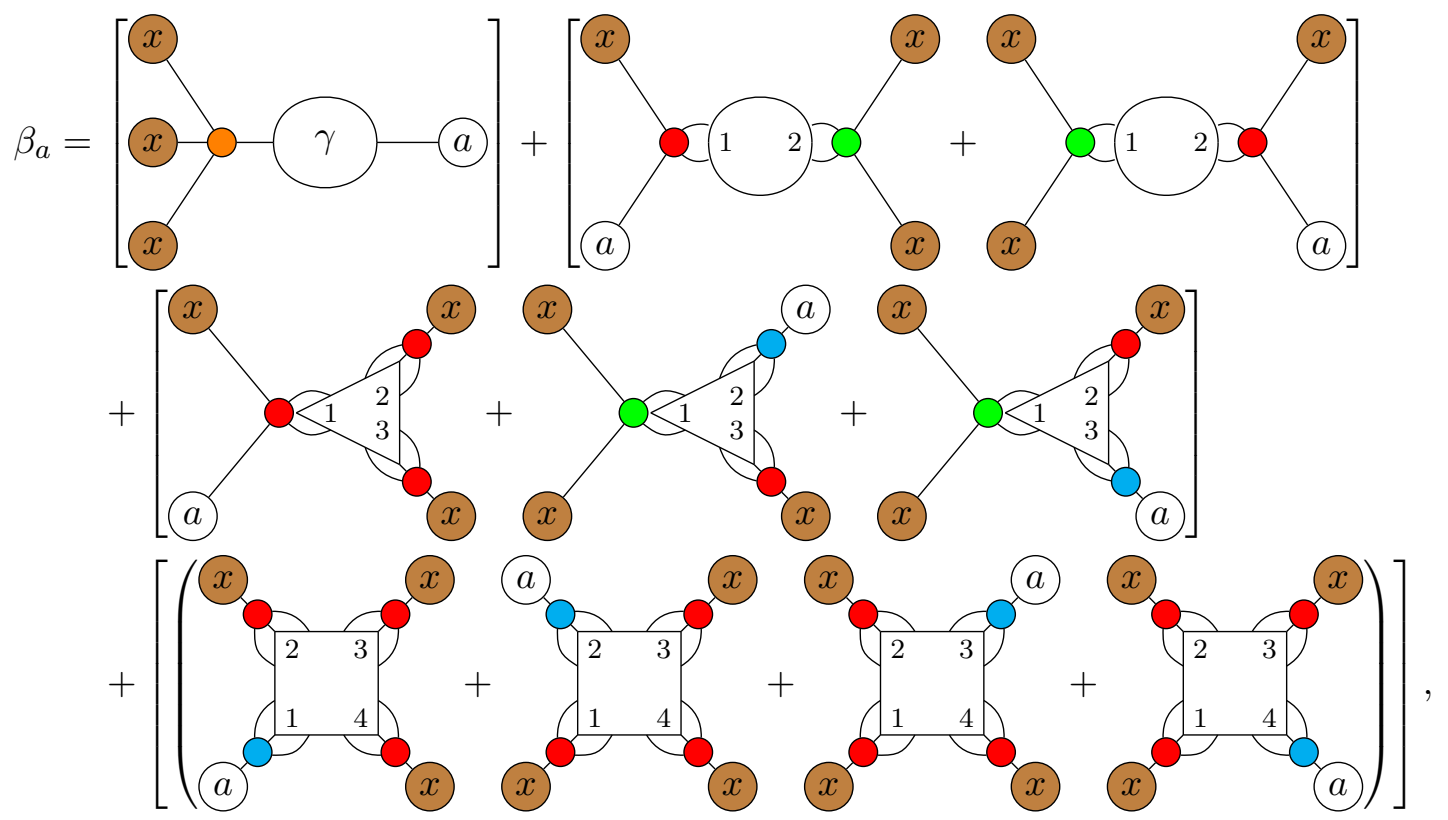

where the orange vertex corresponds the tadpole parameter $t_{a}$ of the Lagrangian (1.1). The analytic form of eq. (A.7) looks like

$$
\begin{aligned}
\beta_{a}= & t_{f} \gamma_{f b}^{\phi}+h_{a e f} m_{g h}^{2}\left[\bigcirc_{e f \mid g h}+\bigcirc_{g h \mid e f}\right] \\
& +\left[h_{a e f} h_{g h i} h_{j k l} \triangle_{e f|g h i| j k l}+m_{e f}^{2} \lambda_{a g h i} h_{j k l}\left(\triangle_{e f|g h i| j k l}+\triangle_{e f|j k l| g h i}\right)\right] \\
& +\lambda_{a e f g} h_{h i j} h_{k l m} h_{\text {nop }}\left[\square_{e f g|h i j| k l m \mid n o p}+\square_{\text {nop } \mid \text { ef } g|h i j| k l m}\right. \\
& \left.+\square_{k l m|n o p| e f g \mid h i j}+\square_{\text {hij|klm|nop|efg }}\right] .
\end{aligned}
$$

\footnotetext{
${ }^{15}$ We correct a couple of misprints in the corresponding expression in the published version of ref. [19].
} 
One more contraction with the dummy field $x_{a}$ gives the beta function of the vacuum energy:

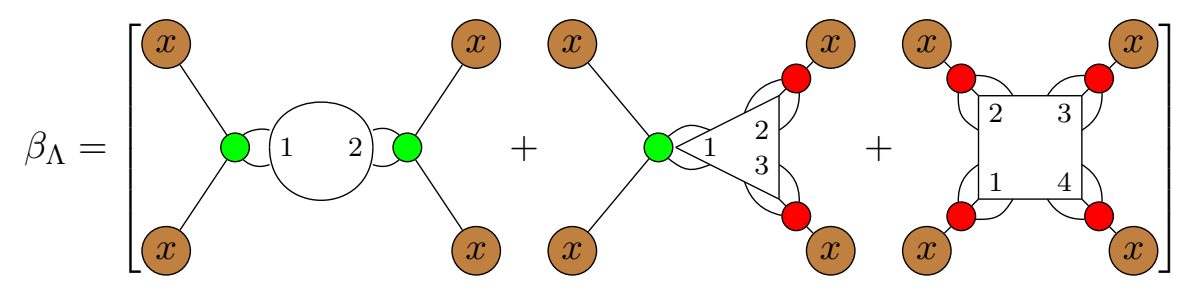

corresponding to

$$
\beta_{\Lambda}=m_{e f}^{2} m_{g h}^{2} \bigcirc_{e f \mid g h}+m_{e f}^{2} h_{g h i} h_{j k l} \triangle_{e f|g h i| j k l}+h_{e f g} h_{h i j} h_{k l m} h_{n o p} \square_{e f g|h i j| k l m \mid n o p} .
$$

Loop expansion of the structures

$$
\begin{aligned}
\bigcirc_{a b \mid c d} & =\sum_{l=1}^{6} \bigcirc_{a b \mid c d}^{(l)}, \\
\triangle_{a b|c d e| f g h} & =\sum_{l=1}^{6} \triangle_{a b|c d e| f g h}^{(l)}, \\
\square_{a b c|d e f| g h i \mid j k l} & =\sum_{l=1}^{6} \square_{a b c|d e f| g h i \mid j k l}^{(l)}
\end{aligned}
$$

can be found in a supplementary .pdf file.

Open Access. This article is distributed under the terms of the Creative Commons Attribution License (CC-BY 4.0), which permits any use, distribution and reproduction in any medium, provided the original author(s) and source are credited.

\section{References}

[1] G. 't Hooft, Dimensional regularization and the renormalization group, Nucl. Phys. B 61 (1973) 455 [InSPIRE].

[2] M.E. Machacek and M.T. Vaughn, Two loop renormalization group equations in a general quantum field theory. 2. Yukawa Couplings, Nucl. Phys. B 236 (1984) 221 [INSPIRE].

[3] M.E. Machacek and M.T. Vaughn, Two loop renormalization group equations in a general quantum field theory. 1. Wave function renormalization, Nucl. Phys. B 222 (1983) 83 [INSPIRE].

[4] M.E. Machacek and M.T. Vaughn, Two loop renormalization group equations in a general quantum field theory. 3. Scalar quartic couplings, Nucl. Phys. B 249 (1985) 70 [INSPIRE].

[5] M.-x. Luo and Y. Xiao, Two loop renormalization group equations in the standard model, Phys. Rev. Lett. 90 (2003) 011601 [hep-ph/0207271] [INSPIRE].

[6] I. Schienbein, F. Staub, T. Steudtner and K. Svirina, Revisiting RGEs for general gauge theories, Nucl. Phys. B 939 (2019) 1 [arXiv:1809.06797] [InSPIRE].

[7] L.N. Mihaila, J. Salomon and M. Steinhauser, Gauge coupling $\beta$-functions in the standard model to three loops, Phys. Rev. Lett. 108 (2012) 151602 [arXiv:1201.5868] [INSPIRE]. 
[8] A.V. Bednyakov, A.F. Pikelner and V.N. Velizhanin, Anomalous dimensions of gauge fields and gauge coupling $\beta$-functions in the standard model at three loops, JHEP 01 (2013) 017 [arXiv: 1210.6873] [INSPIRE].

[9] A.V. Bednyakov, A.F. Pikelner and V.N. Velizhanin, Yukawa coupling $\beta$-functions in the Standard Model at three loops, Phys. Lett. B 722 (2013) 336 [arXiv:1212.6829] [INSPIRE].

[10] K.G. Chetyrkin and M.F. Zoller, $\beta$-function for the Higgs self-interaction in the Standard Model at three-loop level, JHEP 04 (2013) 091 [Erratum ibid. 09 (2013) 155] [arXiv: 1303.2890] [INSPIRE].

[11] A.V. Bednyakov, A.F. Pikelner and V.N. Velizhanin, Higgs self-coupling $\beta$-function in the Standard Model at three loops, Nucl. Phys. B 875 (2013) 552 [arXiv:1303.4364] [INSPIRE].

[12] F. Herren, L. Mihaila and M. Steinhauser, Gauge and Yukawa coupling $\beta$-functions of two-Higgs-doublet models to three-loop order, Phys. Rev. D 97 (2018) 015016 [Erratum ibid. 101 (2020) 079903] [arXiv:1712.06614] [INSPIRE].

[13] A.V. Bednyakov, On three-loop RGE for the Higgs sector of 2HDM, JHEP 11 (2018) 154 [arXiv: 1809.04527] [INSPIRE].

[14] A.V. Bednyakov and A.F. Pikelner, Four-loop strong coupling $\beta$-function in the standard model, Phys. Lett. B 762 (2016) 151 [arXiv:1508.02680] [inSPIRE].

[15] M.F. Zoller, Top-Yukawa effects on the $\beta$-function of the strong coupling in the SM at four-loop level, JHEP 02 (2016) 095 [arXiv: 1508.03624] [INSPIRE].

[16] K.G. Chetyrkin and M.F. Zoller, Leading QCD-induced four-loop contributions to the $\beta$-function of the Higgs self-coupling in the SM and vacuum stability, JHEP 06 (2016) 175 [arXiv: 1604.00853] [INSPIRE].

[17] J. Davies, F. Herren, C. Poole, M. Steinhauser and A.E. Thomsen, Gauge coupling $\beta$ functions to four-loop order in the standard model, Phys. Rev. Lett. 124 (2020) 071803 [arXiv: 1912.07624] [INSPIRE].

[18] C. Poole and A.E. Thomsen, Constraints on 3- and 4-loop $\beta$-functions in a general four-dimensional quantum field theory, JHEP 09 (2019) 055 [arXiv: 1906.04625] [INSPIRE].

[19] T. Steudtner, General scalar renormalisation group equations at three-loop order, JHEP 12 (2020) 012 [arXiv: 2007.06591] [INSPIRE].

[20] T. Steudtner, Towards general scalar-Yukawa renormalisation group equations at three-loop order, arXiv:2101.05823 [INSPIRE].

[21] A.E. Thomsen, RGBeta: a Mathematica package for the evaluation of renormalization group $\beta$-functions, arXiv:2101.08265 [INSPIRE].

[22] M.V. Kompaniets and E. Panzer, Minimally subtracted six loop renormalization of $\mathrm{O}(n)$-symmetric $\phi^{4}$ theory and critical exponents, Phys. Rev. D 96 (2017) 036016 [arXiv: 1705. 06483] [INSPIRE].

[23] A.V. Bednyakov, A.F. Pikelner and V.N. Velizhanin, Three-loop SM $\beta$-functions for matrix Yukawa couplings, Phys. Lett. B 737 (2014) 129 [arXiv:1406.7171] [INSPIRE].

[24] O. Schnetz, Numbers and functions in quantum field theory, Phys. Rev. D 97 (2018) 085018 [arXiv:1606.08598] [INSPIRE].

[25] L.T. Adzhemyan, E.V. Ivanova, M.V. Kompaniets, A. Kudlis and A.I. Sokolov, Six-loop $\varepsilon$ expansion study of three-dimensional n-vector model with cubic anisotropy, Nucl. Phys. B 940 (2019) 332 [arXiv: 1901.02754] [inSPIRE]. 
[26] M.V. Kompaniets, A. Kudlis and A.I. Sokolov, Six-loop $\epsilon$ expansion study of three-dimensional $\mathrm{O}(n) \times \mathrm{O}(m)$ spin models, Nucl. Phys. B 950 (2020) 114874 [arXiv: 1911.01091] [INSPIRE].

[27] M. Tentyukov and J. Fleischer, A Feynman diagram analyzer DIANA, Comput. Phys. Commun. 132 (2000) 124 [hep-ph/9904258] [INSPIRE].

[28] J.A.M. Vermaseren, The symbolic manipulation program FORM.

[29] B. Nickel, D. Meiron, and G. Baker Jr., Compilation of 2-pt and 4-pt graphs for continuous spin model, University of Guelph report (1977).

[30] D. Batkovich, Y. Kirienko, M. Kompaniets and S. Novikov, GraphState - A tool for graph identification and labelling, arXiv:1409.8227 [INSPIRE].

[31] S.P. Martin and M.T. Vaughn, Two loop renormalization group equations for soft supersymmetry breaking couplings, Phys. Rev. D 50 (1994) 2282 [Erratum ibid. 78 (2008) 039903] [hep-ph/9311340] [INSPIRE].

[32] M.-x. Luo, H.-w. Wang and Y. Xiao, Two loop renormalization group equations in general gauge field theories, Phys. Rev. D 67 (2003) 065019 [hep-ph/0211440] [InSPIRE].

[33] M. Kompaniets and K.J. Wiese, Fractal dimension of critical curves in the $O(n)$-symmetric $\phi^{4}$ model and crossover exponent at 6-loop order: loop-erased random walks, self-avoiding walks, Ising, XY, and Heisenberg models, Phys. Rev. E 101 (2020) 012104 [arXiv: 1908. 07502] [INSPIRE].

[34] J.E. Kirkham, Calculation of crossover exponent from Heisenberg to Ising behavior using the fourth order epsilon expansion, J. Phys. A 14 (1981) L437.

[35] B.M. Kastening, Five loop vacuum energy $\beta$-function in phi**4 theory with $O(N)$ symmetric and cubic interactions, Phys. Rev. D 57 (1998) 3567 [hep-ph/9710346] [INSPIRE].

[36] S.A. Larin, M. Monnigmann, M. Strosser and V. Dohm, Five-loop additive renormalization in the $\phi^{4}$ theory and amplitude functions of the minimally renormalized specific heat in three dimensions, Phys. Rev. B 58 (1998) 3394 [cond-mat/9711069] [INSPIRE].

[37] D.F. Litim and T. Steudtner, ARGES - Advanced Renormalisation Group Equation Simplifier, arXiv: 2012.12955 [INSPIRE].

[38] N.V. Antonov, M.V. Kompaniets and N.M. Lebedev, Critical behaviour of the $O(n) \phi^{4}$ model with an antisymmetric tensor order parameter, J. Phys. A 46 (2013) 405002 [arXiv: 1307.1991] [INSPIRE].

[39] N.V. Antonov, M.V. Kompaniets and N.M. Lebedev, Critical behavior of the $\mathrm{O}(n) \phi^{4}$ model with an antisymmetric tensor order parameter: three-loop approximation, Theor. Math. Phys. 190 (2017) 204 [Teor. Mat. Fiz. 190 (2017) 239].

[40] N.M. Lebedev and M.V. Kompaniets, Critical behaviour of a $\mathrm{O}(n)$-symmetric model with antisymmetric tensor order parameter: four-loop approximation, Vestnik SPbSU Phys. Chem. 5 (2018) 20.

[41] P. Calabrese and P. Parruccini, Five loop $\epsilon$-expansion for $\mathrm{O}(n) \times \mathrm{O}(m)$ spin models, Nucl. Phys. B 679 (2004) 568 [cond-mat/0308037] [INSPIRE].

[42] M. De Prato, A. Pelissetto and E. Vicari, Spin-density-wave order in cuprates, Phys. Rev. B 74 (2006) 144507 [cond-mat/0601404] [INSPIRE].

[43] A. Pelissetto and E. Vicari, High-order perturbative expansions of multi-parameter Phi**4 quantum field theories, arXiv:0712.2377 [INSPIRE]. 
[44] M. V. Komarova, M.Y. Nalimov and J. Honkonen, Temperature Green's functions in fermi systems: The superconducting phase transition, Theor. Math. Phys. 176 (2013) 906.

[45] G.A. Kalagov, M.V. Kompaniets and M.Y. Nalimov, Renormalization-group investigation of a superconducting $U(r)$-phase transition using five loops calculations, Nucl. Phys. B 905 (2016) 16 [arXiv:1505.07360] [INSPIRE].

[46] P. Calabrese and P. Parruccini, Five loop $\epsilon$-expansion for $\mathrm{U}(n) \times \mathrm{U}(m)$ models: finite temperature phase transition in light QCD, JHEP 05 (2004) 018 [hep-ph/0403140] [INSPIRE].

[47] M. Hnatic, G.A. Kalagov, and M.Yu. Nalimov, On the first-order phase transition in $\mathrm{SU}(N)$ matrix models, Nucl. Phys. B 955 (2020) 115060.

[48] H. Ruegg, Extremas of SU(N) Higgs potentials and symmetry breaking pattern, Phys. Rev. D 22 (1980) 2040 [inSPIRE].

[49] T. Murphy and L. O'Raifeartaigh, Effect of the renormalization group on the symmetry breaking patterns of SU(n) Higgs potentials, Nucl. Phys. B 229 (1983) 509.

[50] P. Cvitanovic, Group theory for Feynman diagrams in non-Abelian gauge theories, Phys. Rev. D 14 (1976) 1536 [INSPIRE].

[51] S. Giombi, I.R. Klebanov and G. Tarnopolsky, Bosonic tensor models at large $N$ and small $\epsilon$, Phys. Rev. D 96 (2017) 106014 [arXiv:1707.03866] [InSPIRE].

[52] G.C. Branco, P.M. Ferreira, L. Lavoura, M.N. Rebelo, M. Sher and J.P. Silva, Theory and phenomenology of two-Higgs-doublet models, Phys. Rept. 516 (2012) 1 [arXiv:1106.0034] [INSPIRE].

[53] I.P. Ivanov, Building and testing models with extended Higgs sectors, Prog. Part. Nucl. Phys. 95 (2017) 160 [arXiv:1702.03776] [INSPIRE].

[54] A. Trautner, Systematic construction of basis invariants in the 2HDM, JHEP 05 (2019) 208 [arXiv: 1812.02614] [INSPIRE].

[55] F. Staub, SARAH 4: a tool for (not only SUSY) model builders, Comput. Phys. Commun. 185 (2014) 1773 [arXiv: 1309.7223] [InSPIRE].

[56] L. Sartore and I. Schienbein, PyR@TE 3, Comput. Phys. Commun. 261 (2021) 107819 [arXiv: 2007.12700] [INSPIRE].

[57] T. Deppisch and F. Herren, RGE++: A C++ library to solve renormalisation group equations in quantum field theory, arXiv:2101.00021 [INSPIRE].

[58] S.P. Martin, Effective potential at three loops, Phys. Rev. D 96 (2017) 096005 [arXiv: 1709.02397] [INSPIRE].

[59] A.V. Manohar and E. Nardoni, Renormalization group improvement of the effective potential: an EFT approach, JHEP 04 (2021) 093 [arXiv: 2010.15806] [INSPIRE]. 